\section{Research Square}

\title{
Emerging SARS-CoV-2 variants expand species tropism to rodents
}

\section{Huiping Shuai}

University of Hong Kong

Jasper Chan

The University of Hong Kong https://orcid.org/0000-0001-6336-6657

\section{Terrence Tsz-Tai Yuen}

University of Hong Kong

\section{Chaemin Yoon}

The University of Hong Kong

Jingchu Hu

Shenzhen Institutes of Advanced Technology https://orcid.org/0000-0001-6144-7226

\section{Lei Wen}

University of Hong Kong

\section{Bingjie Hu}

University of Hong Kong

\section{Dong Yang}

University of Hong Kong

\section{Yixin Wang}

University of Hong Kong

\section{Yuxin Hou}

University of Hong Kong

\section{Xiner Huang}

The University of Hong Kong https://orcid.org/0000-0002-0154-8372

\section{Yue Chai}

University of Hong Kong

\section{Chris Chan}

The University of Hong Kong https://orcid.org/0000-0001-7089-4829

\section{Vincent Poon}

The University of Hong Kong

\section{Rui-Qi Zhang}

University of Hong Kong

\section{Wan-Mui Chan}

University of Hong Kong

Jonathan Daniel Ip 
University of Hong Kong

\section{Allen Wing Ho Chu}

University of Hong Kong

\section{Ye-fan Hu}

University of Hong Kong

Jian-Piao Cai

University of Hong Kong

\section{Kwok-Hung Chan}

The University of Hong Kong

\section{Jie Zhou}

University of Hong Kong https://orcid.org/0000-0002-2948-3873

\section{Siddharth Sridhar}

The University of Hong Kong

\section{Bao-Zhong Zhang}

University of Hong Kong

\section{Shuofeng Yuan}

University of Hong Kong https://orcid.org/0000-0001-7996-1119

\section{Jinxia Zhang}

Department of microbiology https://orcid.org/0000-0002-5087-3614

\section{Jiandong Huang}

The University of Hong Kong https://orcid.org/0000-0001-7531-2816

\section{Kelvin To}

The University of Hong Kong https://orcid.org/0000-0002-1921-5824

\section{Kwok-Yung Yuen}

The University of Hong Kong https://orcid.org/0000-0002-2083-1552

\section{Hin Chu ( $\square$ hinchu@hku.hk )}

The University of Hong Kong https://orcid.org/0000-0003-2855-9837

\section{Article}

Keywords: SARS-CoV-2 variants, rodents, ACE2

Posted Date: July 13th, 2021

DOI: https://doi.org/10.21203/rs.3.rs-676992/v1

License: (c) (1) This work is licensed under a Creative Commons Attribution 4.0 International License. Read Full License 
1 Title:

2 Emerging SARS-CoV-2 variants expand species tropism to rodents

Authors:

5 Huiping Shuai ${ }^{1,2,3, *}$, Jasper Fuk-Woo Chan ${ }^{1,2,3,4,5, \#, *}$, Terrence Tsz-Tai Yuen ${ }^{2, *}$, Chaemin

6 Yoon $^{2}$, Jing-Chu $\mathrm{Hu}^{6}$, Lei Wen², Bingjie $\mathrm{Hu}^{2}$, Dong Yang ${ }^{2}$, Yixin Wang ${ }^{2}$, Yuxin $\mathrm{Hou}^{2}$, Xiner

7 Huang $^{2}$, Yue Chai ${ }^{2}$, Chris Chung-Sing Chan ${ }^{2}$, Vincent Kwok-Man Poon ${ }^{2}$, Rui-Qi Zhang ${ }^{2}$,

8 Wan-Mui Chan ${ }^{2}$, Jonathan Daniel $\mathrm{Ip}^{2}$, Allen Wing-Ho $\mathrm{Chu}^{2}$, Ye-Fan $\mathrm{Hu}^{7}$, Jian-Piao Cai ${ }^{2}$,

9 Kwok-Hung Chan ${ }^{1,2,3}$, Jie Zhou ${ }^{1,2,3}$, Siddharth Sridhar ${ }^{1,2,3}$, Bao-Zhong Zhang ${ }^{6}$, Shuofeng

10 Yuan ${ }^{1,2,3}$, Anna Jinxia Zhang ${ }^{1,2,3}$, Jian-Dong Huang ${ }^{6,7}$, Kelvin Kai-Wang To ${ }^{1,2,3,5}$, Kwok-

11 Yung Yuen ${ }^{1,2,3,4,5, \#}$, Hin $\mathrm{Chu}^{1,2,3, \#}$

Affiliations:

${ }^{1}$ State Key Laboratory of Emerging Infectious Diseases, Li Ka Shing Faculty of Medicine,

The University of Hong Kong, Pokfulam, Hong Kong Special Administrative Region, China.

${ }^{2}$ Department of Microbiology, Li Ka Shing Faculty of Medicine, The University of Hong

Kong, Pokfulam, Hong Kong Special Administrative Region, China

${ }^{3} \mathrm{Carol} \mathrm{Yu}$ Centre for Infection, Li Ka Shing Faculty of Medicine, The University of Hong

Kong, Pokfulam, Hong Kong Special Administrative Region, China

${ }^{4}$ Department of Microbiology, Queen Mary Hospital, Pokfulam, Hong Kong Special

Administrative Region, China

${ }^{5}$ Hainan Medical University-The University of Hong Kong Joint Laboratory of Tropical 
$25{ }^{6}$ Synthetic Biology, Shenzhen Institutes of Advanced Technology, Chinese Academy of

26 Sciences; Shenzhen, People's Republic of China

$27{ }^{7}$ School of Biomedical Sciences, Li Ka Shing Faculty of Medicine, The University of Hong

28 Kong; Pokfulam, Hong Kong Special Administrative Region, China

$29 *$ These authors contributed equally as co-first authors

30

\#Correspondence: Hin Chu (hinchu@hku.hk), Kwok-Yung Yuen (kyyuen@hku.hk), and Jasper Fuk-Woo Chan (jfwchan@hku.hk). State Key Laboratory of Emerging Infectious

Diseases, Carol Yu Centre for Infection, Department of Microbiology, Li Ka Shing Faculty of Medicine, The University of Hong Kong, Pokfulam, Hong Kong Special Administrative Region, China. Tel: 852-22552402. Fax: 852-28551241. 


\section{Abstract}

Mice are not susceptible to wildtype SARS-CoV-2 infection. Emerging SARS-CoV-2 variants including B.1.1.7, B.1.351, P.1, and P.3 contain mutations in spike, which have been suggested to associate with an increased recognition of mouse ACE2, raising the postulation that they may have evolved to expand species tropism to rodents. Here, we investigated the capacity of B.1.1.7 and other emerging SARS-CoV-2 variants in infecting mouse (Mus musculus) and rats (Rattus norvegicus) under in vitro and in vivo settings. Our results show that B.1.1.7 and P.3, but not B.1 or wildtype SARS-CoV-2, can utilize mouse and rat ACE2 for virus entry in vitro. High infectious virus titers, abundant viral antigen expression, and pathological changes are detected in the nasal turbinate and lung of B.1.1.7-inocluated mice and rats. Together, these results reveal that the current predominant circulating SARS-CoV-2 variant, B.1.1.7, has gained the capability to expand species tropism to rodents. 
Coronaviruses have repeatedly crossed species barriers to cause human infection ${ }^{1}$. Severe acute respiratory syndrome coronavirus 2 (SARS-CoV-2) is a novel lineage B humanpathogenic betacoronavirus that was first identified in December $2019^{2}$. SARS-CoV-2 is highly transmissible and has rapidly spread worldwide to cause more than 180 million cases of Coronavirus Disease 2019 (COVID-19) with over 3.9 million deaths ${ }^{3,4}$. Same as SARSCoV-2 and human coronavirus NL63 (HCoV-NL63), SARS-CoV-2 also uses angiotensinconverting enzyme II (ACE2) as the cellular receptor for entry ${ }^{5}$. Importantly, new SARSCoV-2 variants of concern (VOC) with amino acid substitutions at the receptor-binding domain of spike protein continue to emerge as the pandemic expands and are potentially associated with increased transmissibility and/or virulence ${ }^{6}$.

Mammals, especially bats and rodents, are considered to be the main animal reservoirs of alphacoronaviruses and betacoronaviruses ${ }^{1,7}$. The global distribution, rapid growth rate, high population densities, and close peridomestic relationship with humans of synanthropic rodent species like rats and mice give them important roles in the transmission cycle of various zoonotic pathogens ${ }^{8-10}$. We and others have previously shown that murine cells are not susceptible to SARS-CoV-2 infection in vitro and mice are not infectable by SARS-CoV-2 in vivo $^{11,12}$, which is due to the amino acid substitutions present at the ACE2-spike interacting surface on mouse ACE2 (mACE2) compared with human ACE2 (hACE2) that render SARSCoV-2 spike unable to utilize mACE2 for cell entry ${ }^{13,14}$. Interestingly, emerging SARSCoV-2 variants, including B.1.1.7 ${ }^{6}$, B.1.351 ${ }^{15}$, P. $1^{16}$, and P. $3^{17}$, all contain the N501Y mutation in spike that has been suggested to be associated with mouse adaptation ${ }^{18}$, raising the postulation that these emerging SARS-CoV-2 variants may have evolved to expand species tropism to mouse and possibly other rodents. To evaluate this possibility with major 
public health significance, we in parallel evaluated the capacity of SARS-CoV-2 wildtype (WT) and SARS-CoV-2 B.1.1.7 variant to infect mice (Mus musculus) and rats (Rattus norvegicus). Our results demonstrate that while mice and rats are not permissive to WT SARS-CoV-2, B.1.1.7 efficiently infects these rodents with production of infectious virus particles in the nasal turbinate and lung for 4-to-7 days post infection. These findings indicate that the predominant circulating SARS-CoV-2 variant, B.1.1.7, have gained the capacity to expand species tropism to rodents and that public health control measures including stringent rodent control should be implemented to facilitate the control of the ongoing pandemic.

\section{Results}

\section{The predominant circulating SARS-CoV-2 variant B.1.1.7 efficiently infects mice}

B.1.1.7 emerged in late 2020 and has quickly become the dominant SARS-CoV-2 variant among all recently reported SARS-CoV-2 isolates in countries on different continents (Fig. 1a and Fig. S1). In line with the surge of B.1.1.7, the N501Y mutation in spike, which is one of the B.1.1.7-defining mutations, has emerged as the most predominant spike mutation since late 2020 (Fig. 1b). This is associated with critical public health importance since N501Y has been implicated in an increased recognition of mouse ACE2 ${ }^{18}$, hinting that the naturallyemerging N501Y-carrying SARS-CoV-2 variants, including B.1.1.7, B.1.351, P.1, and P.3, may have evolved to infect rodents. To evaluate this possibility, we assessed SARS-CoV-2 pseudovirus entry in the non-permissive BHK21 cells with mouse ACE2 (mACE2), rat ACE2 (rACE2), or human ACE2 (hACE2) overexpression. Our results demonstrated that BHK21 cells with mACE2 or rACE2 overexpression were permissive to the entry of SARSCoV-2-N501Y-spike pseudoviruses, but not SARS-CoV-2-WT-spike pseudoviruses. In contrast, both pseudoviruses efficiently utilized hACE2 for entry (Fig. 1c). Next, we evaluated the capacity of SARS-CoV-2 variants to infect BHK21 cells expressing mACE2, 
rACE2, or hACE2. As demonstrated in Figure 1d and 1e, SARS-CoV-2 WT and B.1 (Fig. S2), which do not carry N501Y, did not infect or replicate in BHK21 cells overexpressing mACE2 or rACE2. In stark contrast, B.1.1.7 and P.3 (Fig. S2), which carry N501Y, both efficiently infected and replicated in mACE2- or rACE2-expressing BHK21 cells (Fig. 1d,e). The viral gene copies in the cell lysate samples of B.1.1.7-infected BHK21 cells expressing mACE2 or rACE2 were $1880(\mathrm{P}=0.0040)$ and $1623(\mathrm{P}=0.0093)$ folds higher than that of the control (vector-transfected) BHK21 cells, respectively (Fig. 1e). Together, these findings suggest that the recently emerged N501Y-carrying SARS-CoV-2 variants, including B.1.1.7, B.1.351, P.1, and P.3, can utilize murine ACE2 as entry receptor and may infect murine species without the need of further adaptation.

To directly address the question of whether these emerging SARS-CoV-2 variants have evolved to gain the capacity to cross species barrier to murine species, we intranasally challenged 6- to 8-week-old C57B6 mice (Mus musculus) with B.1.1.7 or WT SARS-CoV-2 and collected tissues for virological and histological assessment on 2, 4, and 7 days post infection (d.p.i.) (Fig. 1f). Importantly, in B.1.1.7-inoculated mice, virus gene copies were detected in brain, nasal turbinate, lung, spleen, and colon tissues. In particular, robust virus replication was detected in the nasal turbinate and lung tissues, which were 4044 and 330 folds higher than that of WT SARS-CoV-2-inoculated mice at 2 d.p.i., respectively. Viral gene copies were cleared from WT SARS-CoV-2-inoculated mice at 7 d.p.i., but remained readily detectable from the nasal turbinate and lung of B.1.1.7-incolated mice (Fig. 1g,h). Permissibility to mouse cells was not due to the D614G mutation since B.1 that carries D614G did not infect and replicate in the nasal turbinate and lung of the inoculated mice (Fig. 1i). Importantly, high-titer infectious virus particles were recovered from the nasal turbinate and lung of B.1.1.7-inocluated mice at 2 d.p.i. and remained detectable in some of the 
inoculated animals at 4 d.pi. and 7 d.p.i (Fig. 1j,k). In comparison, infectious viral titers were only marginally detected in the nasal turbinates of mice inoculated with WT SARS-CoV-2 at 2 d.p.i. but not at 4 d.p.i. and 7 d.p.i.. No infectious virus particle could be recovered from the lung of WT SARS-CoV-2-inoculated mice. Together, these findings indicate that the predominant circulating SARS-CoV-2 variant, B.1.1.7, but not WT SARS-CoV-2, can infect mice with robust virus shedding from the nasal turbinate.

\section{Mice (Mus musculus) develop pathological changes after primary B.1.1.7 inoculation}

Next, we evaluated viral antigen expression and virus-induced pathology in the respiratory tract of mice upon B.1.1.7 or WT SARS-CoV-2 inoculation. As demonstrated in Figure 2a, abundant viral nucleocapsid $(\mathrm{N})$ protein expression was detected in mice inoculated with B.1.1.7 but not WT SARS-CoV-2. In the nasal turbinate, robust viral N protein expression was detected in the epithelial lining at 2 d.p.i. (Fig. 2a) and remained detectable at 4 d.p.i. (Fig. S3). In the lung, abundant $\mathrm{N}$ protein was detected in the bronchioles and alveoli (Fig. $2 b)$. Viral $N$ protein in the lung of B.1.1.7-inoculated mice was most readily detected at 2 d.p.i. and remained detectable at 7 d.p.i. (Fig. S3). In contrast to B.1.1.7-inoculated mice, no viral $\mathrm{N}$ protein could be detected in the nasal turbinate and lung of WT SARS-CoV-2incoluated mice (Fig. 2a,b and Fig. S3). Histological examination of B.1.1.7-inoculated mice showed epithelial damage with secretion and cell debris in the nasal turbinate (Fig. 2c, 2 d.p.i., red arrows) at 2 d.p.i.. At 4 d.p.i., more severe submucosal infiltration and loss of epithelial integrity were observed in multiple locations (Fig. 2c, 4 d.p.i., red arrows). At 7 d.p.i., intact nasal turbinate epithelium with resolved inflammatory infiltration was observed in the B.1.1.7-inoculated mice, indicating regeneration of the epithelial lining after virus infection (Fig. 2c, 7 d.p.i., red arrow). In mice inoculated with WT SARS-CoV-2, the nasal turbinate epithelium remained intact from 2 d.p.i. to 7 d.p.i. (Fig. 2c, blue arrows). In line 
with the histopathological findings of the nasal turbinate, the lung of B.1.1.7-inoculated mice showed alveolar wall congestion and inflammatory infiltration (Fig. 2d, 2 d.p.i, red arrows) accompanied by alveolar haemorrhage (Fig. 2d, 4 d.p.i, red arrows). Perivascular infiltration and alveolar septal thickening in the lung of B.1.1.7-inoculated mice remained evident at 7 d.p.i (Fig. 2d, 7 d.p.i, red arrows). In contrast, the lung of WT SARS-CoV-2-inoculated mice showed no histopathological changes at all examined time points (Fig. 2d, blue arrows). Despite the histopathological changes found in B.1.1.7-inoculated mice, no significant body weight loss was observed (Fig. S4).

Moreover, we rechallenged B.1.1.7- or WT SARS-CoV-2-incoluated mice at 14 d.p.i. with B.1.1.7 and harvested their nasal turbinate, lung, and blood samples at 14 days post rechallenge (d.p.r.). Our results showed that mice previously inoculated with B.1.1.7 but not WT SARS-CoV-2 were partially protected from virus rechallenge as evidenced by the lower virus gene copy detected in the nasal turbinate and lung (Fig. 2f). In agreement with the observed partial protection, we detected the presence of serum SARS-CoV-2-specific neutralizing antibodies at 14 days post primary virus challenge in B.1.1.7-incoluated but not WT SARS-CoV-2-inoculated mice (Fig. 2g). The level of neutralizing antibody was further enhanced upon rechallenge, in a virus inoculum-dependent manner (Fig. 2h). Taken together, our results demonstrate that the current predominant circulating B.1.1.7 variant has expanded species tropism to mice with high infectious virus titer shedding from their nasal turbinate.

\section{B.1.1.7 infects and causes pathological damages in rats (Rattus norvegicus)}

Brown rats or street rats live in close proximity to humans and play key roles in disseminating zoonotic diseases. Rat ACE2 (rACE2) is similar to mACE2 in amino acid sequences (Fig. S5) and supported B.1.1.7 entry in vitro (Fig. 1c-e). To evaluate whether rats 
are susceptible to B.1.1.7 infection, we challenged 6-week-old Sprague Dawley rats (Rattus norvegicus) intranasally with B.1.1.7 or WT SARS-CoV-2 (Fig. 3a). Similar to B.1.1.7 infection in mice, the viral gene copies of the inoculated rats peaked at 2 d.p.i. and were 17.0and 1,831.7-folds higher in the nasal turbinate and lung, respectively, than that of WT SARSCoV-2-inoculated rats (Fig. 3b,c). Importantly, infectious virus titers were recovered from the nasal turbinate and lung of B.1.1.7-inoculated but not WT SARS-CoV-2-inoculated rats, indicating that rats were permissive to B.1.1.7 but not WT SARS-CoV-2 (Fig. d,e). In line with the infectious virus titer results, immunohistochemistry staining revealed that viral $\mathrm{N}$ protein was more frequently detected in the lung than the nasal turbinate of B.1.1.7inoculated rats (Fig. 3f), while viral $\mathrm{N}$ protein was absent in the lung and nasal turbinate of WT SARS-CoV-2-inoculated rats (Fig. 3f).

In the nasal turbinate of B.1.1.7-inoculated rats, histological examination showed loss of mucosal integrity and epithelial damage at 2 d.p.i. (Fig. 3g, 2 d.p.i., red arrows). More severe epithelial sloughing and submucosal infiltration were detected at 4 d.p.i. (Fig. $3 g, 4$ d.p.i., red arrow), which were largely regenerated at 7 d.p.i. (Fig. $3 g, 7$ d.p.i., red arrow). In contrast, the epithelial lining in the nasal turbinate of WT SARS-CoV-2-inoculated rats remained intact at all time points examined (Fig. 3g, blue arrows). In the lung of B.1.1.7-inoculated rats, moderate to severe multi-focal consolidation in the alveoli was observed at 2 d.p.i.. (Fig. 3h, 2 d.p.i., red arrows). Moreover, necrotic cell debris in the bronchioles, perivascular cuffing caused by inflammatory infiltration, fibrin deposition in the interstitium, and alveolar septa thickening were also observed (Fig. 3i). Alveolar congestion, epithelial disruption in the bronchioles, and alveoli infiltration remained evident at 4 d.p.i. (Fig. 3h, 4 d.p.i., red arrows). Upon viral clearance at 7 d.p.i., these lung pathologies were largely resolved (Fig. 3 h, 7 d.p.i., red arrows). In contrast to B.1.1.7-inocluated rats, no pathological change was observed in 
the lung of WT SARS-CoV-2-inoculated rats at any examined time points (Fig. 3h, blue arrows). These findings reveal that the current predominant circulating SARS-CoV-2 variant, B.1.1.7, has evolved to expand species tropism beyond WT SARS-CoV-2, and can infect rodents including rats and mice.

\section{Differential SARS-CoV-2 tropism in K18-hACE2 and wild type mice}

Since mice are not susceptible to WT SARS-CoV-2 infection ${ }^{12}$, the K18-hACE2 transgenic mice are utilized as an animal model for SARS-CoV-2 infection ${ }^{19}$. Our current findings reveal that B.1.1.7 can naturally infect mice, which may serve as a new animal model for SARS-CoV-2 infection that does not require prior virus adaptation or introduction of human ACE2 to the mice. To compare the two infection models, we first characterized the physiological distribution of ACE2 in the two mouse models (Fig. 4a). Our data showed that the K18-hACE2 and C57B6 mice expressed comparable levels of mACE2. Mouse ACE2 expression in the intestine was the highest, followed by kidney, colon, lung, and nasal turbinate (Fig. 4b), which resembled the natural ACE2 distribution in human ${ }^{20,21}$. In addition to mACE2, K18-hACE2 mice express high level of hACE2 at all evaluated organs (Fig. 4c). The hACE2 expression level was 3,719.1-, 28.3-, 1.1-, 369.2-, 8,285.5-, 1,035.8-, 26.0-, 37.1, and 36.0-folds higher than that of the mACE2 expression in brain, nasal turbinate, lung, heart, liver, spleen, small intestine, colon, and kidney of K18-hACE2 mice, respectively (Fig. $4 b, c)$.

Next, we in parallel inoculated 6- to 8-week-old C57B6 and K18-hACE2 mice with B.1.1.7 and evaluated virus replication at 2 d.p.i.. Interestingly, our results indicated that B.1.1.7 replication was more robust in the nasal turbinate of C57B6 mice than that of the K18hACE2 mice [(vRNA; C57B6: $2.15 \times 10^{6}$ vs K18-hACE2: $\left.2.17 \times 10^{4} ; \mathrm{p}=0.0234\right)$ and (PFU; 
C57B6: $9.87 \times 10^{5}$ vs K18-hACE2: $\left.1.50 \times 10^{5} ; \mathrm{p}=0.0014\right]$. In contrast, B.1.1.7 replication was

more robust in the lungs of K18-hACE2 mice than that of the C57B6 mice [(vRNA; C57B6: 1.08 $\times 10^{4} / 10^{3}$ HPRT vs K18-hACE2: $\left.5.36 \times 10^{6} / 10^{3} \mathrm{HPRT} ; \mathrm{p}=0.0005\right)$ and (PFU; C57B6: $7.67 \times 10^{4} /$ gram tissue vs K18-hACE2: $1.02 \times 10^{7} /$ gram tissue; $\left.\mathrm{p}<0.0001\right]$ (Fig. $4 \mathrm{~d}, \mathrm{e}$ ). These findings were validated by immunohistochemistry staining, which consistently detected more abundant viral $\mathrm{N}$ protein expression in the nasal turbinate of C57B6 mice than K18-ACE2 mice. Similarly, viral N protein was most frequently and abundantly detected in the lung of K18-hACE2 mice than C57B6 mice (Fig. 4f). In addition to the nasal turbinate and lung, high levels of infectious virus titer and viral antigen expression were detected in the brain of K18hACE2 mice, which is suggested to cause fatal outcome in the infected animals ${ }^{22}$. In contrast, the brain of C57B6 mice was free of either infectious virus particles or viral antigen expression (Fig. 4e,f). In addition to evaluating B.1.1.7 infection in C57B6 and K18-hACE2 mice, we additionally compared the infection of B.1.1.7 and WT SARS-CoV-2 in K18hACE2 mice. Our results indicated that the B.1.1.7 and WT SARS-CoV-2 have similar replication efficiency in the nasal turbinate and lung of K18-hACE2 mice (Fig. S6). Taken together, the B.1.1.7-inoculated C57B6 mouse model may represent a more physiologically relevant model for studying SARS-CoV-2 pathogenesis and therapeutics evaluation due to its native ACE2 distribution profile, while the K18-hACE2 mouse model will be useful for studying the central nervous system pathologies of SARS-CoV-2 infection.

\section{Discussion}

In summary, we demonstrate that the current dominant circulating SARS-CoV-2 variant, B.1.1.7, has evolved to gain the capability to cross species barrier to infect rodents including mice and rats, which are not permissive to WT SARS-CoV-2. Mice and rats reside in close proximity to humans, including densely populated areas with circulating B.1.1.7 and/or other 
251 N501Y-carrying SARS-CoV-2 variants. The acquired susceptibility of these rodents to

SARS-CoV-2 variants has substantially increased the risk of SARS-CoV-2 infection in the rodent population, which may result in a zoonotic reservoir of SARS-CoV-2. In particular, our results indicate that B.1.1.7-infected mice and rats can shed infectious virus particles in the respiratory tract for approximately 4 to 7 days after infection, which may contaminate the environment and become a persistent source of human infection. Importantly, SARS-CoV-2 adaptation in mice occurs rapidly, with mouse-adapting mutations usually developing within 1-to-10 passages ${ }^{18,23-25}$. These mutations will result in even more robust SARS-CoV-2 replication in rodents and may further facilitate virus dissemination.

Interspecies spill-over of SARS-CoV-2 is not uncommon. While SARS-CoV-2 is presumed to have originated from an animal reservoir, humans are also known to infect other animals with SARS-CoV-2, including cats, dogs, and even tigers in zoos ${ }^{26-28}$. A previous study demonstrated that bidirectional SARS-CoV-2 transmission could occur between humans and minks ${ }^{29}$. Rodents including mice and rats were previously not permissive to SARS-CoV-2 infection and were thus not considered to be an important animal reservoir of the ongoing COVID-19 pandemic. However, as demonstrated in the current study, the evolving SARSCoV-2 variants with the N501Y mutation in spike can now naturally infect mice and rats, and potentially other rodent species. Considering the abundance of these animals and their proximate habitats with humans, humans-to-rodents and rodents-to-humans transmissions may be inevitable. Public health measures including stringent rodent control and surveillance on SARS-CoV-2 infection in the rodent populations especially in densely populated regions should be implemented to facilitate the control of the pandemic.

\section{Methods}


All virus strains used in this study were shown in Figure S2. SARS-CoV-2 wildtype virus HKU-001a (GenBank: MT230904) was a clinical isolate as previously described ${ }^{11}$. SARSCoV-2 B.1 (GISAID: EPI_ISL_497840), B.1.1.7 (GISAID: EPI_ISL_1273444), and B.1.1.28.3 (P.3) (GISAID: EPI_ISL_1660475) were isolated from laboratory-confirmed COVID-19 patients in Hong Kong. In vitro and in vivo experiments involving infectious wild type SARS-CoV-2, B.1, B.1.1.7, and P.3 were performed in Biosafety Level 3 laboratory and strictly followed the approved standard operation procedures.

\section{SARS-CoV-2-Spike-pseudovirus entry assay}

The SARS-CoV-2-WT-S and SARS-CoV-2-N501Y-S pseudoviruses were generated in 293T cells as we previously described ${ }^{30}$. For pseudovirus entry assays, mouse ACE2, rat ACE2, or human ACE2 were transfected into BHK21 cells. At 24-hour post transfection, transfected cells were inoculated with pseudoviruses for $24 \mathrm{~h}$ before washed and lysed for measuring luciferase activity with a firefly luciferase assay system (E1501, Promega).

\section{In vivo virus challenge}

The use of animals was approved by the Committee on the Use of Live Animals in Teaching and Research of The University of Hong Kong. Heterogenous K18-hACE2 C57BL/6J mice (2B6.Cg-Tg(K18-ACE2)2Prlmn/J) were obtained from The Jackson Laboratory. For virus challenge in mice, 6- to 8- week-old female C57BL/6J mice or K18-hACE2 transgenic mice were anaesthetized with ketamine and xylazine, followed by intranasal inoculation with 20 $\mu 1 /$ mouse of WT, B.1, or B.1.1.7 SARS-CoV-2 at $4 \times 10^{3}$ PFU/mouse as we previously described ${ }^{31}$. For SARS-CoV-2 infection in rats, 6-week-old female Sprague Dawley rats were anaesthetized with ketamine and xylazine, followed by intranasal inoculation with 
$4 \times 10^{5} \mathrm{PFU} /$ rat of WT or B.1.1.7 SARS-CoV-2 diluted in 50 $\mu 1$ PBS. On day 2-, 4- and 7-day post virus challenge, animals were euthanized for harvesting organs for viral load titration and histology staining.

\section{RNA extraction and quantitative RT-PCR}

RNA extraction and RT-qPCR were performed as we previously described ${ }^{11}$. SARS-CoV-2 genome copies were quantified with sequence-specific probe and primers targeting the RNAdependent RNA polymerase ${ }^{11}$.

\section{Infectious virus titration by plaque assays}

Organs harvested from virus-inoculated animals were homogenized in DMEM with Tissue Lyzer II, followed by centrifugation and titration. To titrate the infectious virus titer, tissue homogenates were 10-fold serially-diluted with DMEM and applied to monolayered VeroE6 cells for $2 \mathrm{~h}$. After inoculation, cells were washed once before overlaid with $1 \%$ low-melting agarose containing 1\% FBS. Cells were further incubated for $72 \mathrm{~h}$ and fixed with $0.5 \%$ crystal violet for visualizing plaque formation ${ }^{32}$.

\section{Histology and immunohistochemistry staining}

Animal tissues were harvested and fixed with $10 \%$ neutral-buffered formalin. Nasal turbinates were decalcified with $10 \%$ formic acid for 7 days before being processed with the TP1020 Leica semi-enclosed benchtop tissue processor. IHC was performed with the DAB (3,3'-diaminobenzidine) substrate kit (Vector Laboratories) as we previously described ${ }^{33}$. To detect the viral antigen, in-house mouse monoclonal biotinylated anti-SARS-CoV-2 nucleocapsid protein antibody was used, followed by color development with the DAB substrate kit. The nuclei were detected with haematoxylin before the tissue sections was 
mounted with the VectaMount permanent mounting medium (Vector Laboratories). For H\&E staining, tissue sections were stained with Gill's haematoxylin and eosin-Y. Images were acquired with the Olympus BX53 light microscope.

\section{Immunofluorescence staining}

Nucleocapsid of SARS-CoV-2 was detected by the in-house rabbit polyclonal anti-SARSCoV-2 nucleocapsid antibody as we previously reported ${ }^{34}$. Nuclei were stained with the DAPI dye (Thermo Fisher Scientific) before the tissue sections being mounted with the Diamond Prolong Antifade mounting buffer (Thermo Fisher Scientific). Images were acquired with the Olympus BX53 light microscope.

\section{Neutralizing antibody assay}

Mice serum was obtained immediately before virus rechallenge and 14 days post rechallenge. Sera were heat-inactivated for $30 \mathrm{~m}$ at $56{ }^{\circ} \mathrm{C}$ before use. Two-fold serially diluted sera was incubated with 100 TCID $_{50}$ WT SARS-CoV-2 for $1 \mathrm{~h}$ at $37{ }^{\circ} \mathrm{C}$. After incubation, the virussera mixture was inoculated in VeroE6 cells and further incubated for $72 \mathrm{~h}$ for cytopathic effect (CPE) development. The neutralization titer was determined by observing the highest dilution without CPE.

\section{Multiple sequence alignment and docking}

Sequence alignment and docking were performed as we previously described ${ }^{35}$. ACE2 protein sequences of human (Q9BYF1), mouse (Q8R0I0), and rat (Q5EGZ1) were retrieved from Uniprot. Multiple sequence alignment was constructed with Muscle. The crystal structure of wildtype SARS-CoV-2 spike RBD and human ACE2 complex (code: 6M0J) was retrieved from the Protein Data Bank. Mouse and rat ACE2 structures were generated by 
mutating the interface residues within $4.0 \AA$ of $\mathrm{RBD}$ according to the multiple sequence alignment. The complex of ACE2 and N501Y RBD was built by superimposition of N501Y RBD (code: 7NEG) with wildtype RBD. All ACE2-RBD complexes were relaxed and locally refined with Rosetta Relax and Docking protocols. Interface binding energies were estimated with InterfaceAnalyzer application.

\section{Statistical analysis}

Data analysis were performed with Graphpad prism 8.0. Statistical comparison between different groups was performed by one-way ANOVA, two-way ANOVA, or Student's t-test. Differences were considered statistically significant when $\mathrm{p}<0.05$.

\section{Acknowledgments}

This work was partly supported by the donations of the Shaw Foundation of Hong Kong, the Richard Yu and Carol Yu, Michael Seak-Kan Tong, May Tam Mak Mei Yin, Lee Wan Keung Charity Foundation Limited, Hong Kong Sanatorium \& Hospital, Hui Ming, Hui Hoy and Chow Sin Lan Charity Fund Limited, Chan Yin Chuen Memorial Charitable Foundation, Marina Man-Wai Lee, the Hong Kong Hainan Commercial Association South China Microbiology Research Fund, the Jessie \& George Ho Charitable Foundation, Perfect Shape Medical Limited, Kai Chong Tong, Tse Kam Ming Laurence, Foo Oi Foundation Limited, Betty Hing-Chu Lee, Ping Cham So, and Lo Ying Shek Chi Wai Foundation; Funding from the Hong Kong Health and Medical Research Fund (CID-HKU1-5) of the Food and Health Bureau, Hong Kong Special Administrative Region Government; Innovation and Technology Fund (ITF), the Government of the Hong Kong Special Administrative Region; The Consultancy Service for Enhancing Laboratory Surveillance of Emerging Infectious Diseases and Research Capability on Antimicrobial Resistance for Department of Health of the Hong 
Kong Special Administrative Region Government; The National Program on Key Research Project of China (grant no. 2020YFA0707500 and 2020YFA0707504); Sanming Project of Medicine in Shenzhen, China (SZSM201911014); and the High Level-Hospital Program, Health Commission of Guangdong Province, China. The funding sources had no role in the study design, data collection, analysis, interpretation, or writing of the report.

\section{Author contributions}

HS, JF-WC, K-YY, and HC had roles in the study design, data collection, data analysis, data interpretation, and writing of the manuscript. TT-TY, CY, JCH, LW, BH, DY, YW, YH, XH, YC, CC-SC, VK-MP, R-QZ, W-MC, JDI, AW-HC, -YFH, J-PC, K-HC, JZ, SS, B-ZZ, SY, AJZ, J-DH, and KK-WT had roles in the experiments, data collection, data analysis, and data interpretation. All authors reviewed and approved the final version of the manuscript.

\section{Competing interests}

Authors declare that they have no competing interests.

\section{Materials and correspondence}

Correspondence and material requests should be addressed to Dr. Hin Chu, Prof Kwok-Yung Yuen, or Dr. Jasper Fuk-Woo Chan.

\section{Data availability}

The data that support the findings of this study are available from the corresponding authors upon reasonable request. 
4021 Chan, J. F., To, K. K., Tse, H., Jin, D. Y. \& Yuen, K. Y. Interspecies transmission and emergence of

2 Zhou, P. et al. A pneumonia outbreak associated with a new coronavirus of probable bat origin. Nature 579, 270-273, doi:10.1038/s41586-020-2012-7 (2020).

3 Chan, J. F. et al. A familial cluster of pneumonia associated with the 2019 novel coronavirus indicating person-to-person transmission: a study of a family cluster. Lancet 395, 514-523, doi:10.1016/S01406736(20)30154-9 (2020).

4 WHO. (2021).

5 Hoffmann, M. et al. SARS-CoV-2 Cell Entry Depends on ACE2 and TMPRSS2 and Is Blocked by a Clinically Proven Protease Inhibitor. Cell 181, 271-280 e278, doi:10.1016/j.cell.2020.02.052 (2020).

6 Leung, K., Shum, M. H., Leung, G. M., Lam, T. T. \& Wu, J. T. Early transmissibility assessment of the N501Y mutant strains of SARS-CoV-2 in the United Kingdom, October to November 2020. Euro Surveill 26, doi:10.2807/1560-7917.ES.2020.26.1.2002106 (2021).

7 Lau, S. K. et al. Discovery of a novel coronavirus, China Rattus coronavirus HKU24, from Norway rats supports the murine origin of Betacoronavirus 1 and has implications for the ancestor of Betacoronavirus lineage A. J Virol 89, 3076-3092, doi:10.1128/JVI.02420-14 (2015).

8 Firth, C. et al. Detection of zoonotic pathogens and characterization of novel viruses carried by commensal Rattus norvegicus in New York City. mBio 5, e01933-01914, doi:10.1128/mBio.01933-14 (2014).

9 McFarlane, R., Sleigh, A. \& McMichael, T. Synanthropy of wild mammals as a determinant of emerging infectious diseases in the Asian-Australasian region. Ecohealth 9, 24-35, doi:10.1007/s10393-012-0763-9 (2012).

10 Sridhar, S. et al. Transmission of Rat Hepatitis E Virus Infection to Humans in Hong Kong: A Clinical and Epidemiological Analysis. Hepatology 73, 10-22, doi:10.1002/hep.31138 (2021).

11 Chu, H. et al. Comparative tropism, replication kinetics, and cell damage profiling of SARS-CoV-2 and SARS-CoV with implications for clinical manifestations, transmissibility, and laboratory studies of COVID-19: an observational study. Lancet Microbe 1, e14-e23, doi:10.1016/S2666-5247(20)30004-5 (2020).

12 Bao, L. et al. The pathogenicity of SARS-CoV-2 in hACE2 transgenic mice. Nature 583, 830-833, doi:10.1038/s41586-020-2312-y (2020).

13 Zhai, X. et al. Comparison of Severe Acute Respiratory Syndrome Coronavirus 2 Spike Protein Binding to ACE2 Receptors from Human, Pets, Farm Animals, and Putative Intermediate Hosts. $J$ Virol 94, doi:10.1128/JVI.00831-20 (2020).

14 Zhao, X. et al. Broad and Differential Animal Angiotensin-Converting Enzyme 2 Receptor Usage by SARS-CoV-2. J Virol 94, doi:10.1128/JVI.00940-20 (2020).

15 Houriiyah Tegally, E. W., Marta Giovanetti, Arash Iranzadeh, Vagner Fonseca, Jennifer Giandhari, Deelan Doolabh, Sureshnee Pillay, Emmanuel James San, Nokukhanya Msomi, Koleka Mlisana, Anne von Gottberg, Sibongile Walaza, Mushal Allam, Arshad Ismail, Thabo Mohale, Allison J Glass, Susan Engelbrecht, Gert Van Zyl, Wolfgang Preiser, Francesco Petruccione, Alex Sigal, Diana Hardie, Gert Marais, Marvin Hsiao, Stephen Korsman, Mary-Ann Davies, Lynn Tyers, Innocent Mudau, Denis York, Caroline Maslo, Dominique Goedhals, Shareef Abrahams, Oluwakemi Laguda-Akingba, Arghavan Alisoltani-Dehkordi, Adam Godzik, Constantinos Kurt Wibmer, Bryan Trevor Sewell, José Lourenço, Luiz Carlos Junior Alcantara, Sergei L Kosakovsky Pond, Steven Weaver, Darren Martin, Richard J Lessells, Jinal N Bhiman, Carolyn Williamson, Tulio de Oliveira. Emergence and rapid spread of a new severe acute respiratory syndrome-related coronavirus 2 (SARS-CoV-2) lineage with multiple spike mutations in South Africa. medRxiv, doi:https://doi.org/10.1101/2020.12.21.20248640 (2020).

16 Voloch, C. M. et al. Genomic characterization of a novel SARS-CoV-2 lineage from Rio de Janeiro, Brazil. J Virol, doi:10.1128/JVI.00119-21 (2021).

17 Francis A. Tablizo, K. M. K., Carlo M. Lapid, Marc Jerrone R. Castro, Maria Sofia L. Yangzon, Benedict A. Maralit, Marc Edsel C. Ayes, Eva Maria Cutiongco-de la Paz, Alethea R. De Guzman, Jan Michael C. Yap, Jo-Hannah S. Llames, Shiela Mae M. Araiza, Kris P. Punayan, Irish Coleen A. Asin, Candice Francheska B. Tambaoan, Asia Louisa U. Chong, Karol Sophia Agape R. Padilla, Rianna Patricia S. Cruz, El King D. Morado, Joshua Gregor A. Dizon, Razel Nikka M. Hao, Arianne A. Zamora, Devon Ray Pacial, Juan Antonio R. Magalang, Marissa Alejandria, Celia Carlos, Anna OngLim, Edsel Maurice Salvaña, John Q. Wong, Jaime C. Montoya, Maria Rosario Singh-Vergeire, Cynthia P. Saloma. Genome sequencing and analysis of an emergent SARS-CoV-2 variant 
characterized by multiple spike protein mutations detected from the Central Visayas Region of the Philippines. medRxiv, doi:https://doi.org/10.1101/2021.03.03.21252812 (2021).

$18 \mathrm{Gu}, \mathrm{H}$. et al. Adaptation of SARS-CoV-2 in BALB/c mice for testing vaccine efficacy. Science 369 , 1603-1607, doi:10.1126/science.abc4730 (2020).

19 Zheng, J. et al. COVID-19 treatments and pathogenesis including anosmia in K18-hACE2 mice. Nature 589, 603-607, doi:10.1038/s41586-020-2943-z (2021).

20 Hamming, I. et al. Tissue distribution of ACE2 protein, the functional receptor for SARS coronavirus. A first step in understanding SARS pathogenesis. J Pathol 203, 631-637, doi:10.1002/path.1570 (2004).

21 Hikmet, F. et al. The protein expression profile of ACE2 in human tissues. Mol Syst Biol 16, e9610, doi:10.15252/msb.20209610 (2020).

22 McCray, P. B., Jr. et al. Lethal infection of K18-hACE2 mice infected with severe acute respiratory syndrome coronavirus. $J$ Virol 81, 813-821, doi:10.1128/JVI.02012-06 (2007).

23 Zhang, Y. et al. SARS-CoV-2 rapidly adapts in aged BALB/c mice and induces typical pneumonia. $J$ Virol, doi:10.1128/JVI.02477-20 (2021).

24 Wang, J. et al. Mouse-adapted SARS-CoV-2 replicates efficiently in the upper and lower respiratory tract of BALB/c and C57BL/6J mice. Protein Cell 11, 776-782, doi:10.1007/s13238-020-00767-x (2020).

25 Leist, S. R. et al. A Mouse-Adapted SARS-CoV-2 Induces Acute Lung Injury and Mortality in Standard Laboratory Mice. Cell 183, 1070-1085 e1012, doi:10.1016/j.cell.2020.09.050 (2020). Garigliany, M. et al. SARS-CoV-2 Natural Transmission from Human to Cat, Belgium, March 2020. Emerg Infect Dis 26, 3069-3071, doi:10.3201/eid2612.202223 (2020).

27 Sit, T. H. C. et al. Infection of dogs with SARS-CoV-2. Nature 586, 776-778, doi:10.1038/s41586020-2334-5 (2020).

28 McAloose, D. et al. From People to Panthera: Natural SARS-CoV-2 Infection in Tigers and Lions at the Bronx Zoo. mBio 11, doi:10.1128/mBio.02220-20 (2020).

29 Oude Munnink, B. B. et al. Transmission of SARS-CoV-2 on mink farms between humans and mink and back to humans. Science 371, 172-177, doi:10.1126/science.abe5901 (2021).

30 Chu, H. et al. Host and viral determinants for efficient SARS-CoV-2 infection of the human lung. Nat Commun 12, 134, doi:10.1038/s41467-020-20457-w (2021).

31 Chu, H. et al. Targeting highly pathogenic coronavirus-induced apoptosis reduces viral pathogenesis and disease severity. Sci Adv 7, doi:10.1126/sciadv.abf8577 (2021).

32 Chu, H. et al. Comparative Replication and Immune Activation Profiles of SARS-CoV-2 and SARSCoV in Human Lungs: An Ex Vivo Study With Implications for the Pathogenesis of COVID-19. Clin Infect Dis 71, 1400-1409, doi:10.1093/cid/ciaa410 (2020).

33 Chu, H. et al. SARS-CoV-2 Induces a More Robust Innate Immune Response and Replicates Less Efficiently Than SARS-CoV in the Human Intestines: An Ex Vivo Study With Implications on Pathogenesis of COVID-19. Cell Mol Gastroenterol Hepatol 11, 771-781, doi:10.1016/j.jcmgh.2020.09.017 (2021).

34 Shuai, H. et al. Differential immune activation profile of SARS-CoV-2 and SARS-CoV infection in human lung and intestinal cells: Implications for treatment with IFN-beta and IFN inducer. J Infect 81, e1-e10, doi:10.1016/j.jinf.2020.07.016 (2020).

35 Chan, J. F. et al. Simulation of the Clinical and Pathological Manifestations of Coronavirus Disease 2019 (COVID-19) in a Golden Syrian Hamster Model: Implications for Disease Pathogenesis and Transmissibility. Clin Infect Dis 71, 2428-2446, doi:10.1093/cid/ciaa325 (2020). 
a

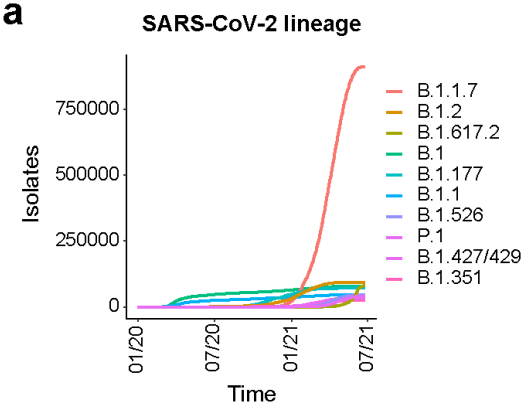

d

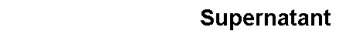

b

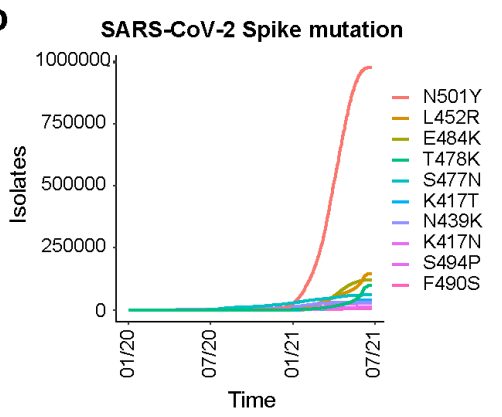

C

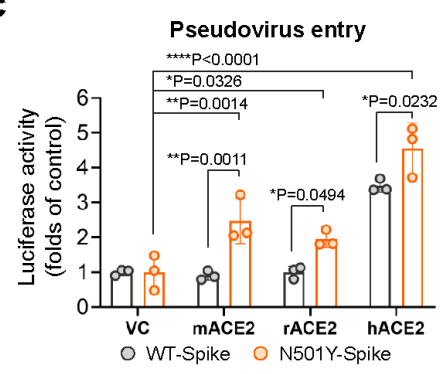

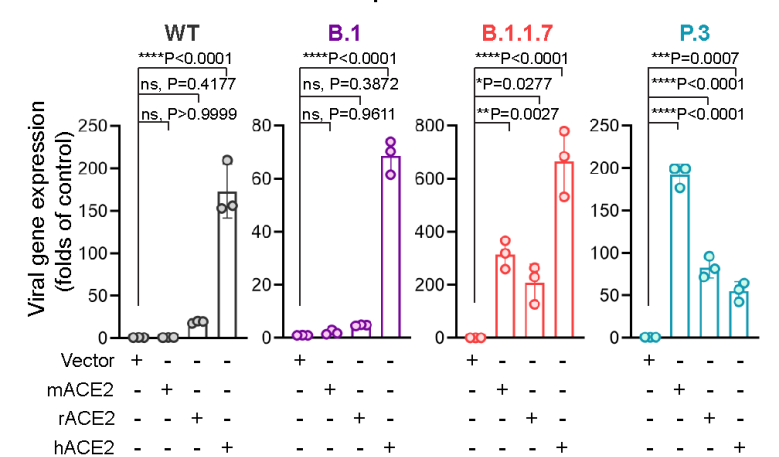

e

Cell lysate

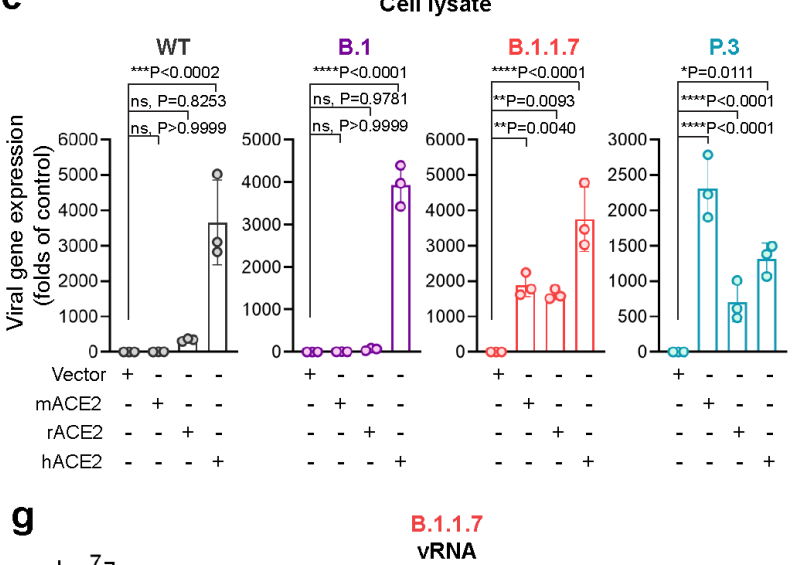

f

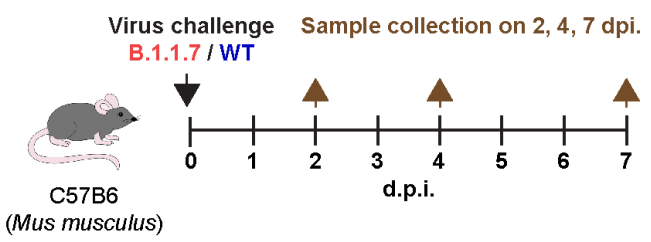

g
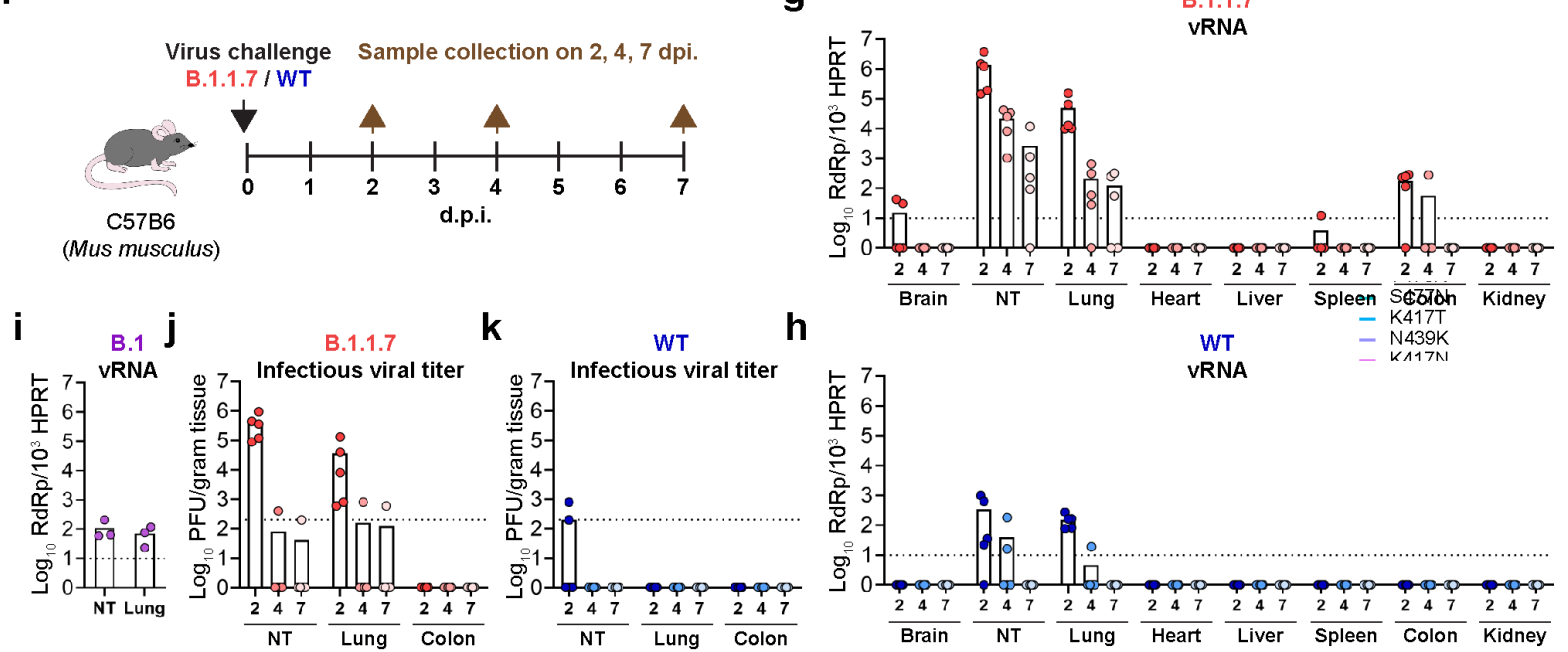

514 Figure 1. The predominant circulating SARS-CoV-2 variant B.1.1.7 efficiently infects

mice. (a) Top 10 prevalent lineages of SARS-CoV-2 and (b) Top 10 amino acid mutations in the spike of SARS-CoV-2 variants retrieved from the GISAID EpiCoV database as of June $28^{\text {th }}, 2021$. (c) BHK21 cells transfected with empty vector, mouse ACE2, rat ACE2 or human ACE2 were inoculated with SARS-CoV-2-S-pseudoviruses with either WT or N501Y spike. Pseudovirus entry was determined at $24 \mathrm{~h}$ post inoculation $(n=3)$. VC, vector control. (d and e) BHK21 cells overexpressing empty vector, mouse ACE, rat ACE2 and human ACE2 were 
infected with B.1, B.1.1.7, P.3, or WT SARS-CoV-2. Relative viral gene expression in (d) supernatants and (e) cell lysates were determined by RT-qPCR $(n=3)$. (f-k) 6-to-8-week-old C57B6 mice were intranasally inoculated with B.1.1.7, B.1, or WT SARS-CoV-2. Mice were euthanized on 2, 4 or 7 d.p.i. and tissues were harvested for viral titer quantification. (f) Schematic illustration of the in vivo infection experiment in C57B6 mice. (g-i) Viral gene copies from mice infected with B.1.1.7, B.1, or WT SARS-CoV-2. ( $\mathrm{j}$ and $\mathrm{k}$ ) Infectious viral titer in the nasal turbinates, lungs and colons of mice inoculated with B.1.1.7 or WT SARSCoV-2. Dashed line indicates detection limits. Data represents mean \pm SD from the indicated number of biological repeats. Statistical differences were determined with two-way analysis of variance (ANOVA) in $(\mathbf{c}),(\mathbf{d})$ and $(\mathbf{e}) .{ }^{*}$ represented $P<0.05 ; * *$ represented $P<0.01$; *** represented $P<0.001 ; * * * *$ represented $P<0.0001$. WT, wild type SARS-CoV-2. NT, nasal turbinate. 
a

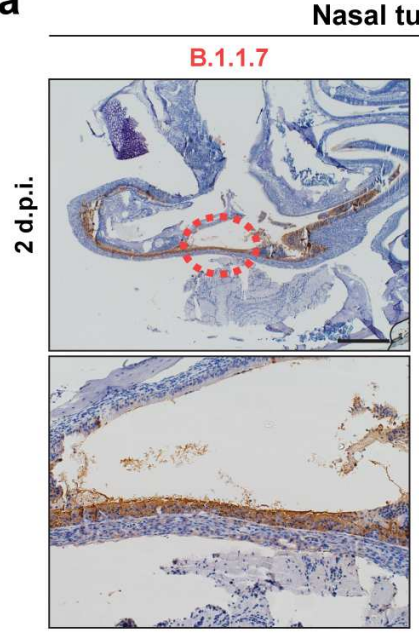

C
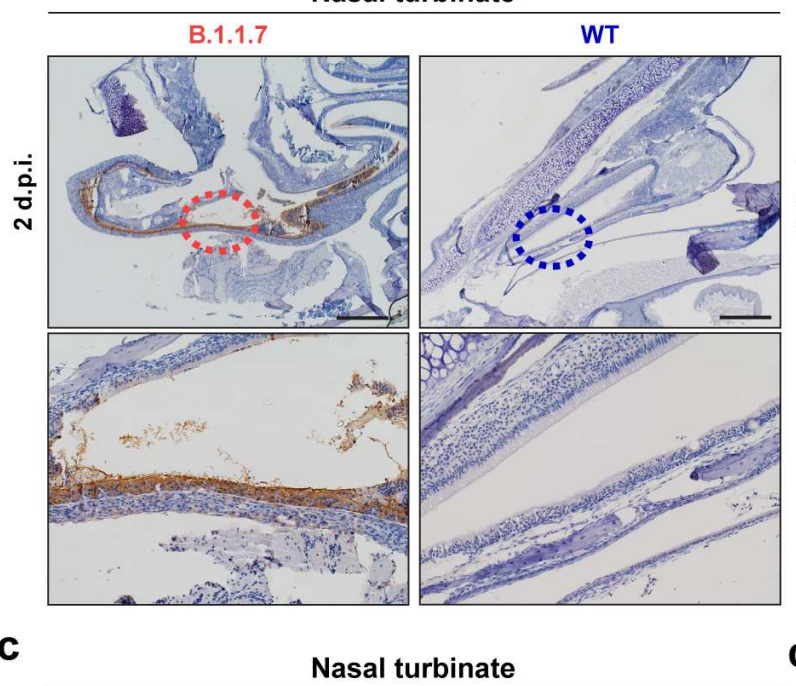

b

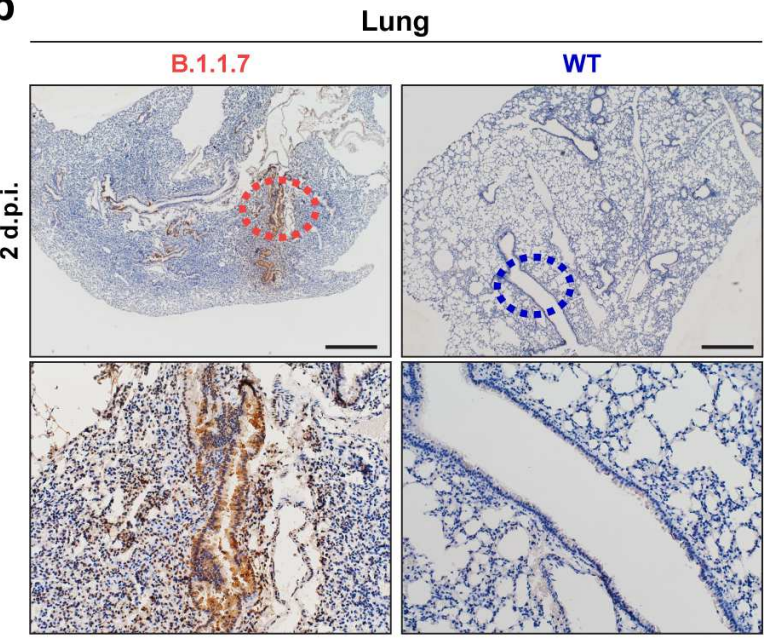

d

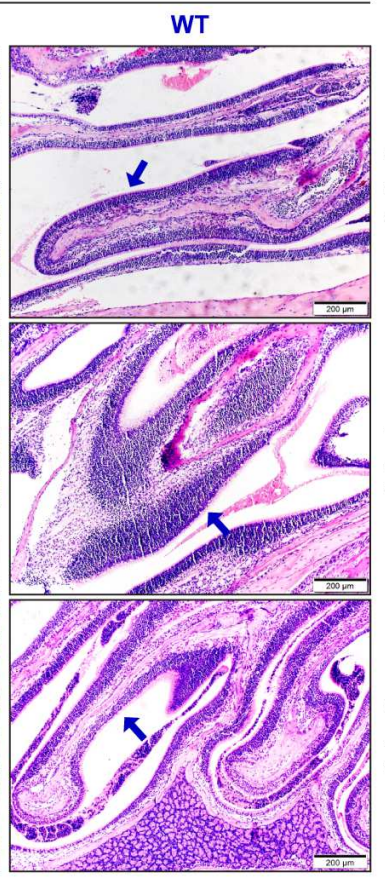

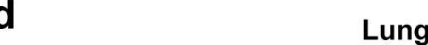

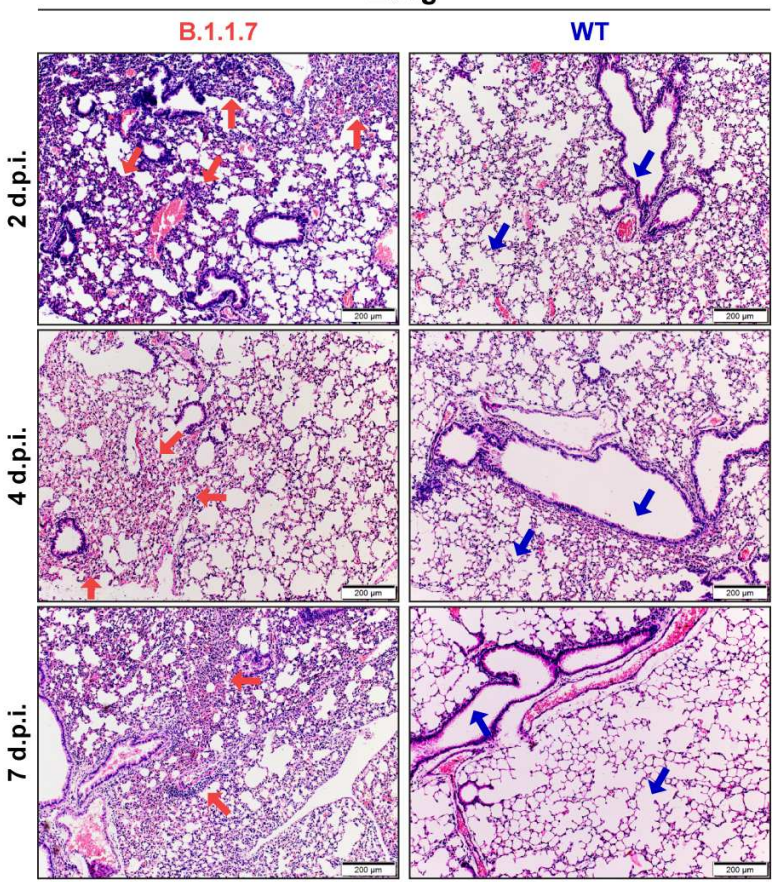

e

Blood Sample

collection

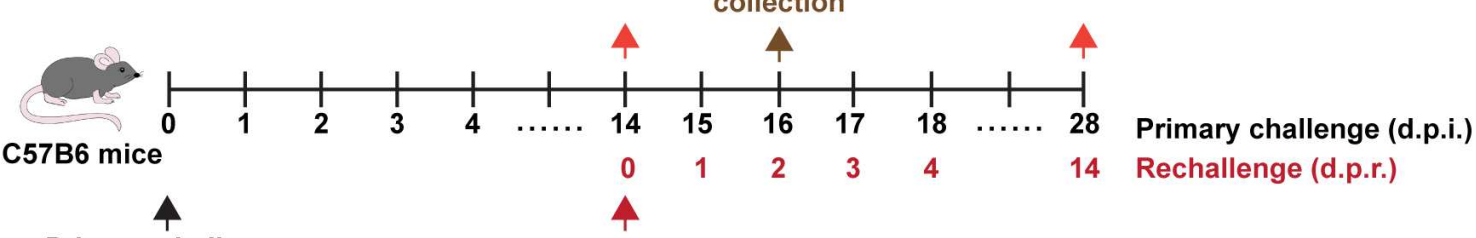

Primary challenge

f

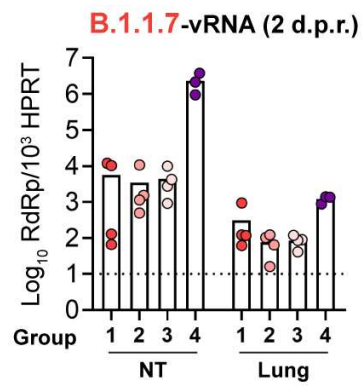

g

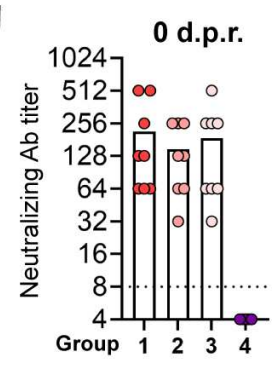

h

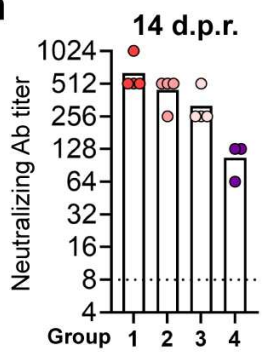

Primary challenge O Group 1: $2 \times 10^{5} \mathrm{PFU}$ B.1.1.7 $\bigcirc$ Group 2: $2 \times 10^{4} \mathrm{PFU}$ O Group 3: $2 \times 10^{3} \mathrm{PFU}$

WT Group 4: 2 × 10 $10^{5} \mathrm{PFU}$ Rechallenge $O$ Group 1: $2 \times 10^{5} \mathrm{PFU}$ B.1.1.7 Group 2: $2 \times 10^{4} \mathrm{PFU}$ Group 3: $2 \times 10^{3} \mathrm{PFU}$
Group $4: 2 \times 10^{5} \mathrm{PFU}$ 
Figure 2. Mice (Mus musculus) develop pathological changes after primary B.1.1.7 inoculation. (a-d) C57B6 mice intranasally inoculated with B.1.1.7 or WT SARS-CoV-2 were euthanized for harvesting nasal turbinate and lung tissues on 2, 4 and 7 d.p.i.. Representative images of immunohistochemistry staining for the detection of nucleocapsid protein (brown) in the (a) nasal turbinates and (b) lung on 2 d.p.i.. Insets were shown below for the circled areas. Scale bar, $500 \mu \mathrm{m}$. (c) Representative images of hematoxylin and eosin (H\&E) staining of mouse nasal turbinates harvested on 2, 4 and 7 d.p.i.. Pathological changes in the B.1.1.7-inoculated mice on 2 d.p.i. and 4 d.p.i. were pointed out with red arrows. On 7 d.p.i., the integrity of the nasal cavity epithelium was restored (red arrow). Intact nasal epithelium layers of the WT SARS-CoV-2-inoculated mice were shown for comparison (blue arrows). (d) Representative images of H\&E staining of mouse lungs harvested on 2, 4 and 7 d.p.i.. In the lungs of B.1.1.7-inoculated mice, alveolar wall congestion and alveolar infiltrations were detected on 2 d.p.i. (red arrows). Alveolar haemorrhage was developed on 4 d.p.i. (red arrows). Alveolar septa thickening remained evident on 7 d.p.i.. Normal structure of bronchioles and alveoli in the lung of the WT SARS-CoV-2-inoculated mice were indicated by blue arrows for comparison. Scale bar, $200 \mu \mathrm{m}$. (e-h) C57B6 mice were intranasally inoculated with $2 \times 10^{5}$ PFU WT SARS-CoV-2 or B.1.1.7 at $2 \times 10^{3}, 2 \times 10^{4}$, or $2 \times 10^{5}$ PFU, followed by rechallenge on 14 d.p.i. with B.1.1.7 at the indicated titers. (e) Schematic illustration of the rechallenge experiment. (f) Viral titer in nasal turbinate and lung of the infected mice on day 2 post rechallenge quantified with RT-qPCR ( $n=4$ for group 1-3, $n=3$ for group 4). (g) Neutralizing antibody titer in mouse sera harvested on 0 day post rechallenge (d.p.r.) $(n=8$ for group 1-3, $n=3$ for group 4$)$ and (h) 14 d.p.r. ( $n=4$ for group $1-3, n=3$ for group 4). Dashed line indicates the detection limit of each assay. NT, nasal turbinate. 
a

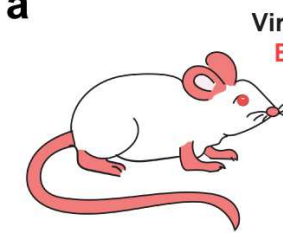

Sprague Dawley rat (Rattus norvegicus)

b

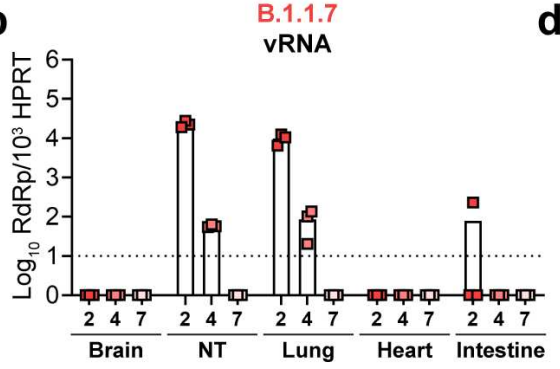

C

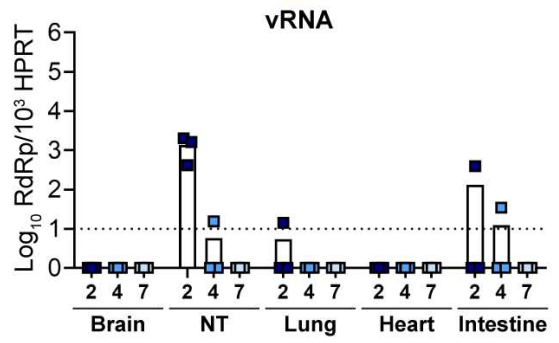

e
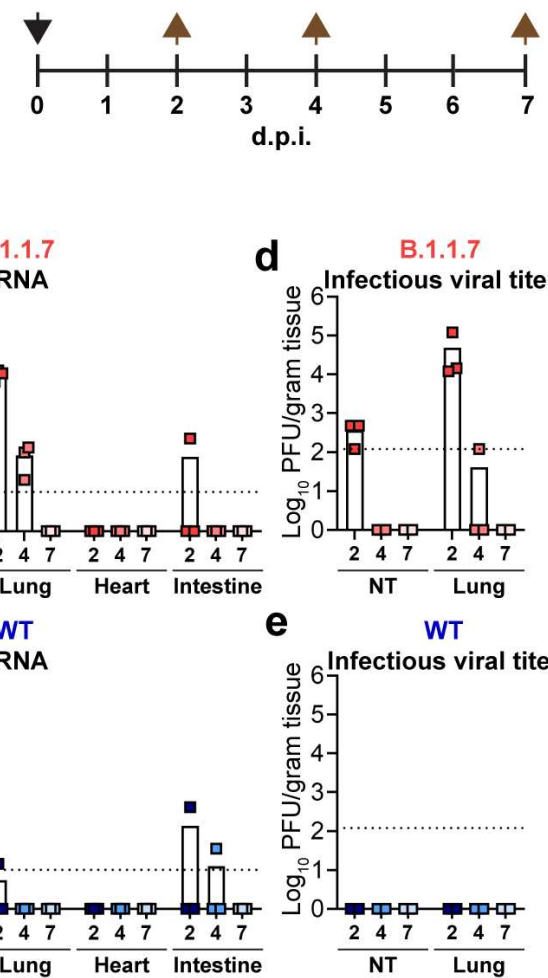

WT f

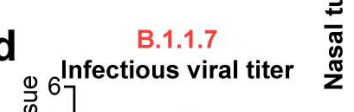

ral titer

\section{导}

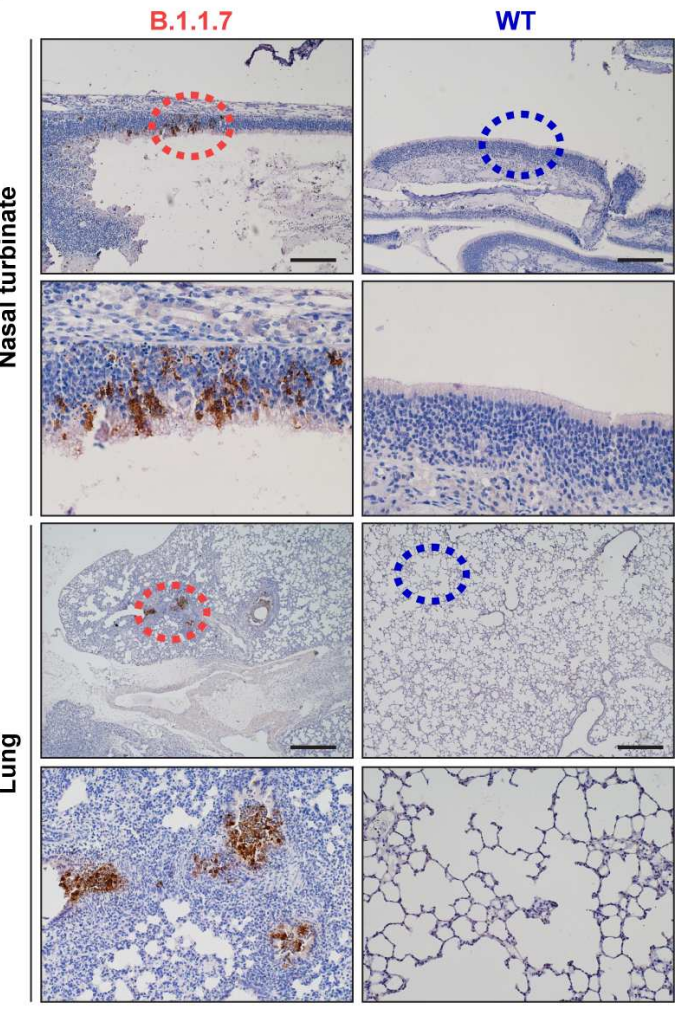

g

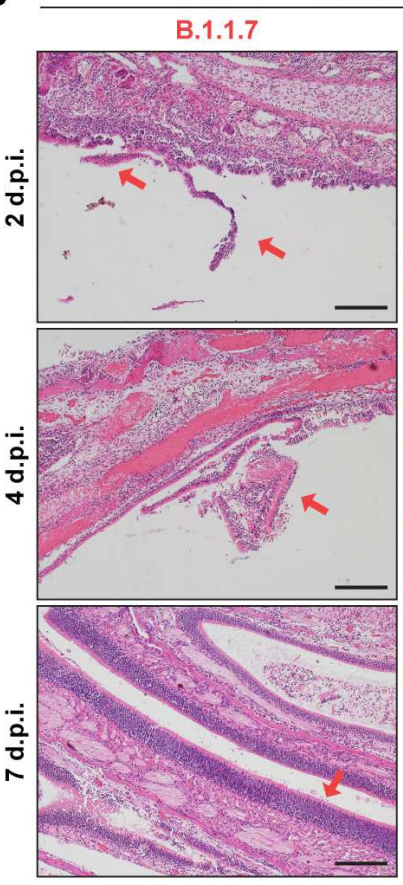

i

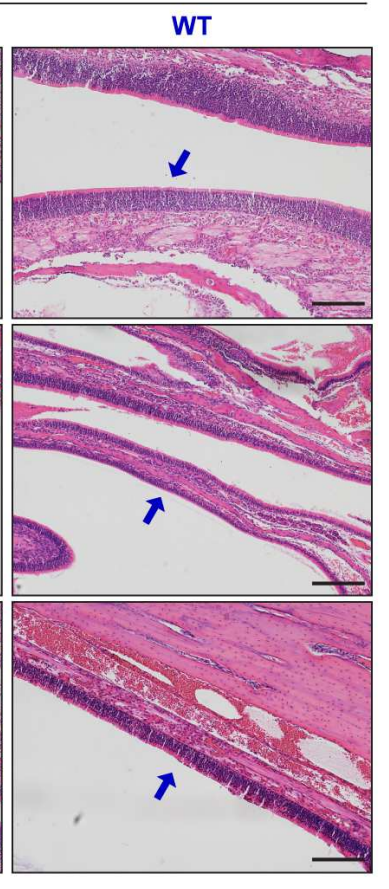
h

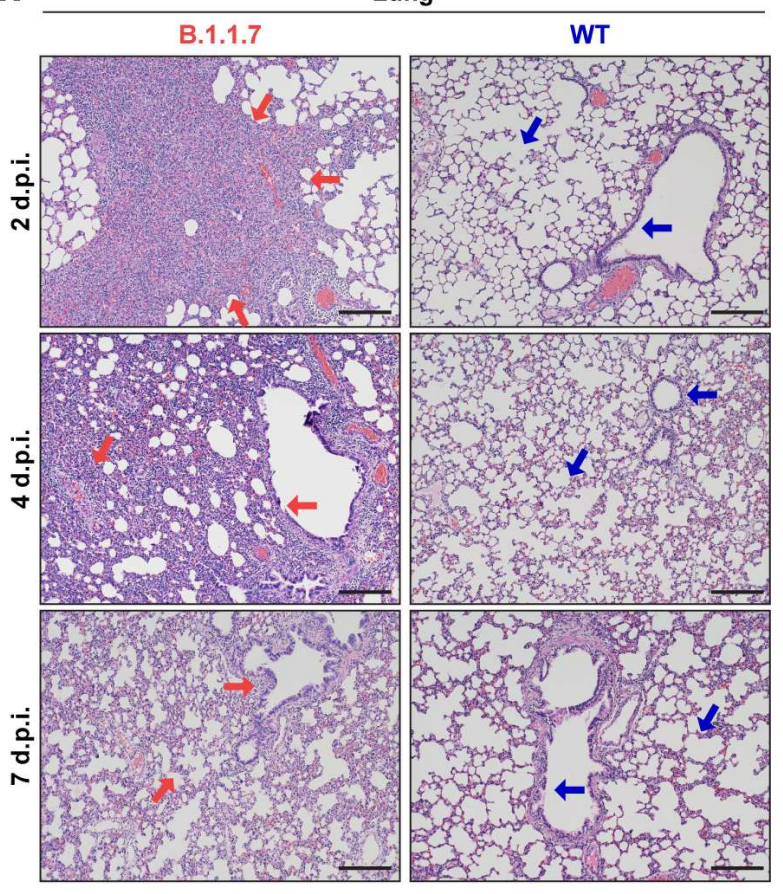

B.1.1.7 Lung 2 d.p.i.
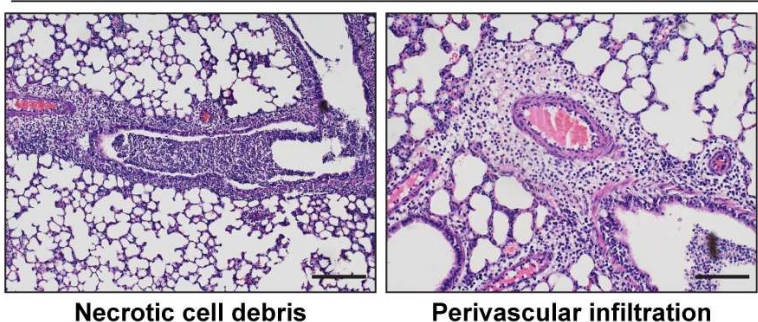

Perivascular infiltration

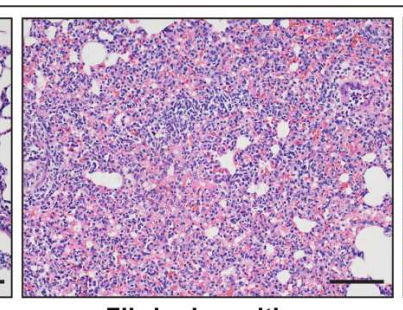

Fibrin deposition

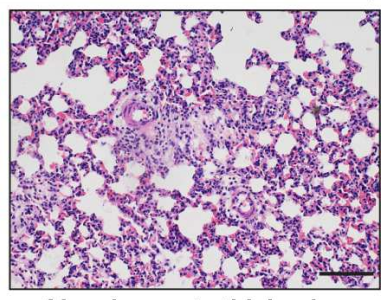

Alveolar septa thickening 
Schematic illustration of in vivo infection experiment in Sprague Dawley rats. 6-week-old Sprague Dawley rats were intranasally inoculated with B.1.1.7 or WT SARS-CoV-2. On 2, 4 and 7 d.p.i., rats were euthanized for harvesting tissues for virological assessment and histopathological analysis. (b and c) Viral titer quantified with RT-qPCR in the brain, nasal turbinate, lung, heart and intestine of the inoculated rats. NT, nasal turbinate. (d and e) Infectious viral titer quantified with plaque assays in the nasal turbinate and lung of the inoculated rats. (f) Immunohistochemistry staining for the detection of nucleocapsid protein expression (brown) in the nasal turbinate and lung of the inoculated rats on 2 d.p.i. Insets were shown below for the circled areas. Scale bar, $200 \mu \mathrm{m}$ for nasal turbinate; $500 \mu \mathrm{m}$ for lung. (g-i) Histopathological analysis for nasal turbinate and lung harvested on 2, 4, and 7 d.p.i.. (g) Representative H\&E images showing pathological changes in the nasal cavity of B.1.1.7-inocuated rats, including epithelial damage and loss of mucosal integrity ( 2 d.p.i., red arrow). On 4 d.p.i., more severe epithelial sloughing and submucosal infiltrations were detected (4 d.p.i., red arrow). On 7 d.p.i., the epithelial lining was largely restored (7 d.p.i., red arrow). In contrast, the structure of the nasal turbinate in WT SARS-CoV-2-inoculated rats remained undisrupted throughout the course of experiment. (h) Representative H\&E images showing severe alveoli consolidation (2 d.p.i., red arrows), epithelium disruption and alveoli infiltrations (4 d.p.i., red arrows) in the lungs of B.1.1.7-inoculated rats. Acute lung injuries were largely repaired by 7 d.p.i. and normal structure of the alveoli and lung were indicated by red arrows. No pathological change was observed in either alveoli or bronchioles of WT SARS-CoV-2-inoculated rats (2, 4, and 7 d.p.i., blue arrows). Scale bar, $200 \mu \mathrm{m}$. (i) Typical pulmonary lesions detected in the lungs of B.1.1.7-inoculated rats on 2 d.p.i.. Scale bar, $100 \mu \mathrm{m}$. 
a

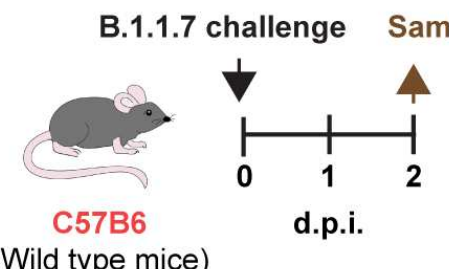

b

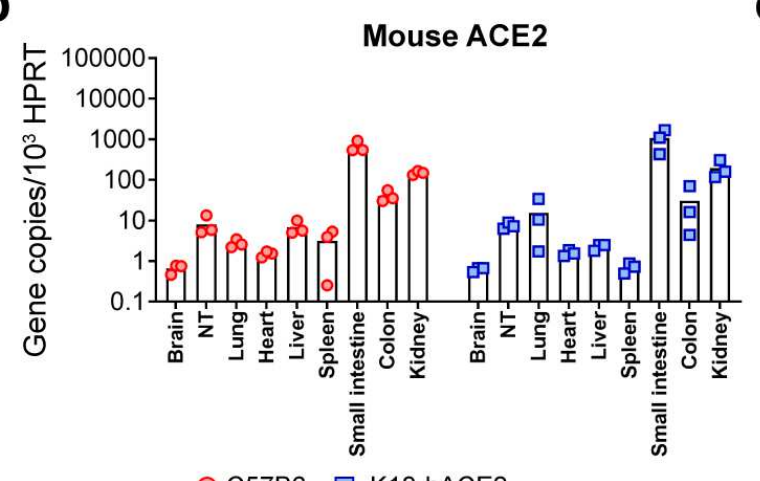

B.1.1.7 challenge Sample collection

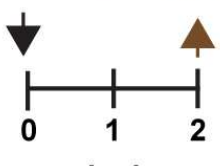

K18-hACE2 (Transgenic mice)

C

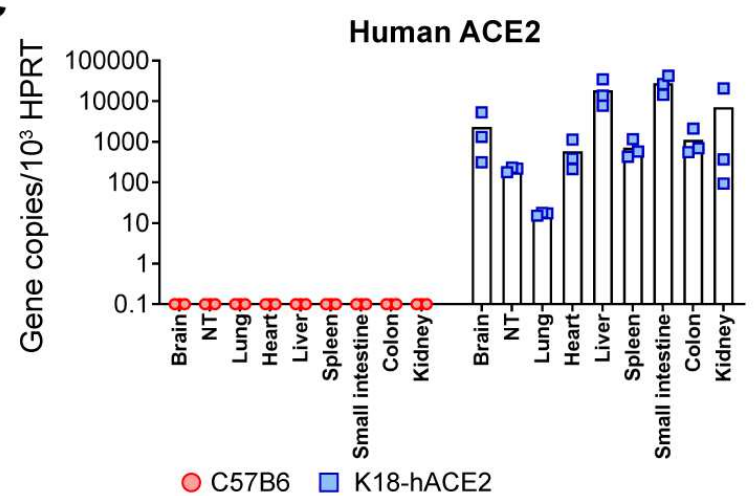

d

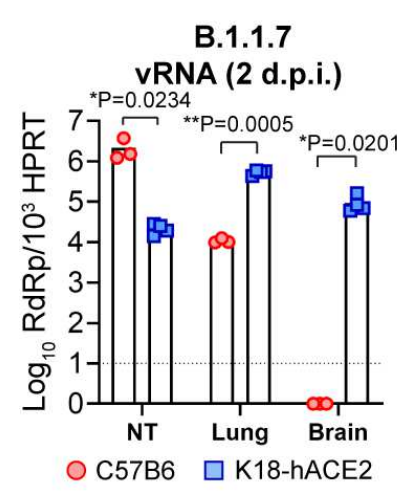

e
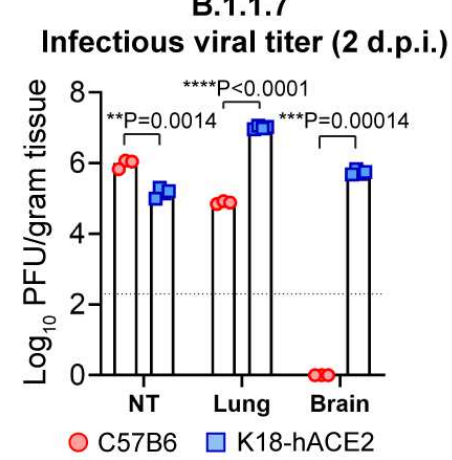

f

B.1.1.7

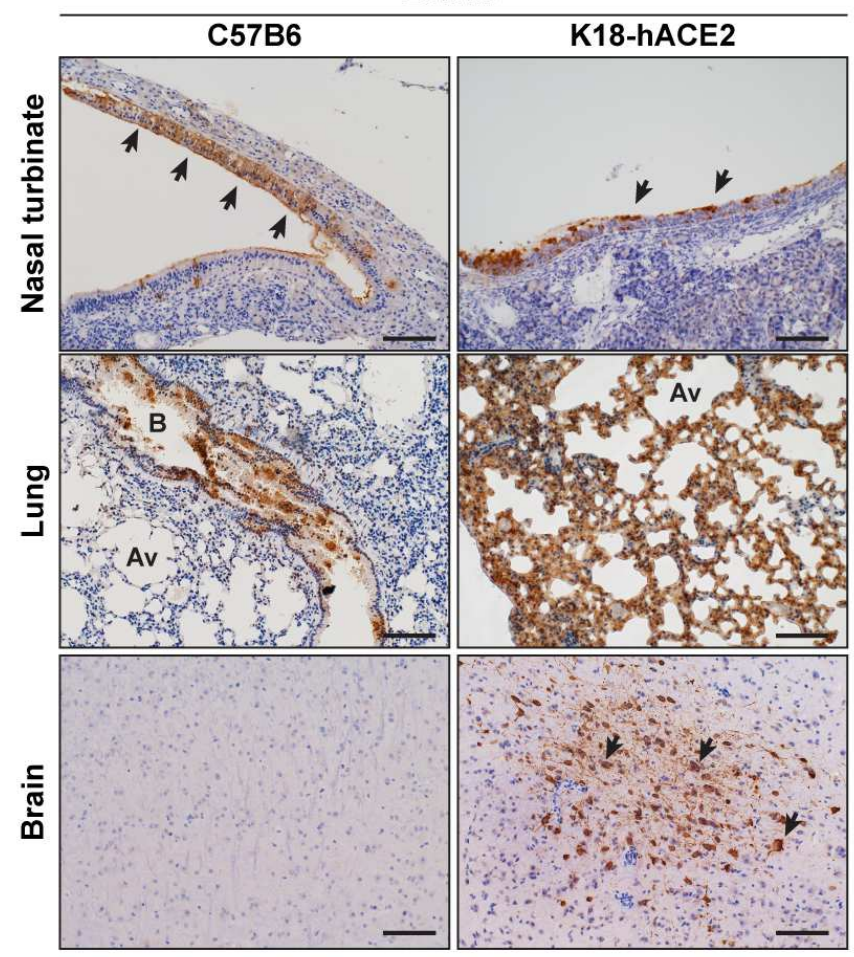

Figure 4. Differential SARS-CoV-2 tropism in K18-hACE2 and wild type mice. (a)

Schematic illustration of comparative study of SARS-CoV-2 B.1.1.7 tropism in C57B6 and expression quantified with RT-qPCR. (d) Viral gene copy in nasal turbinate, lung and brain of the 
591 Infectious viral titers in nasal turbinate, lung, and brain of B.1.1.7-inoculated C57B6 and 592 K18-hACE2 mice quantified with plaque assays. (f) Representative immunohistochemistry 593 images of nasal turbinate, lung, and brain of B.1.1.7-inoculated C57B6 and K18-hACE2 594 mice. Presence of SARS-CoV-2 nucleocapsid protein were shown in brown and indicated by 595 black arrows. Scale bar, $100 \mu \mathrm{m}$. Statistical differences were determined with two-tailed 596 Student's $t$-test in $(\mathbf{d})$ and $(\mathbf{e}) .{ }^{*}$ represented $P<0.05$; ** represented $P<0.01$; *** 597 represented $P<0.001$; **** represented $P<0.0001$. Av, alveoli; B, bronchioles. NT, nasal 598 turbinates; SI, small intestine.

599

600

601

602

603

604

605

606

607

608

609

610

611

612

613 


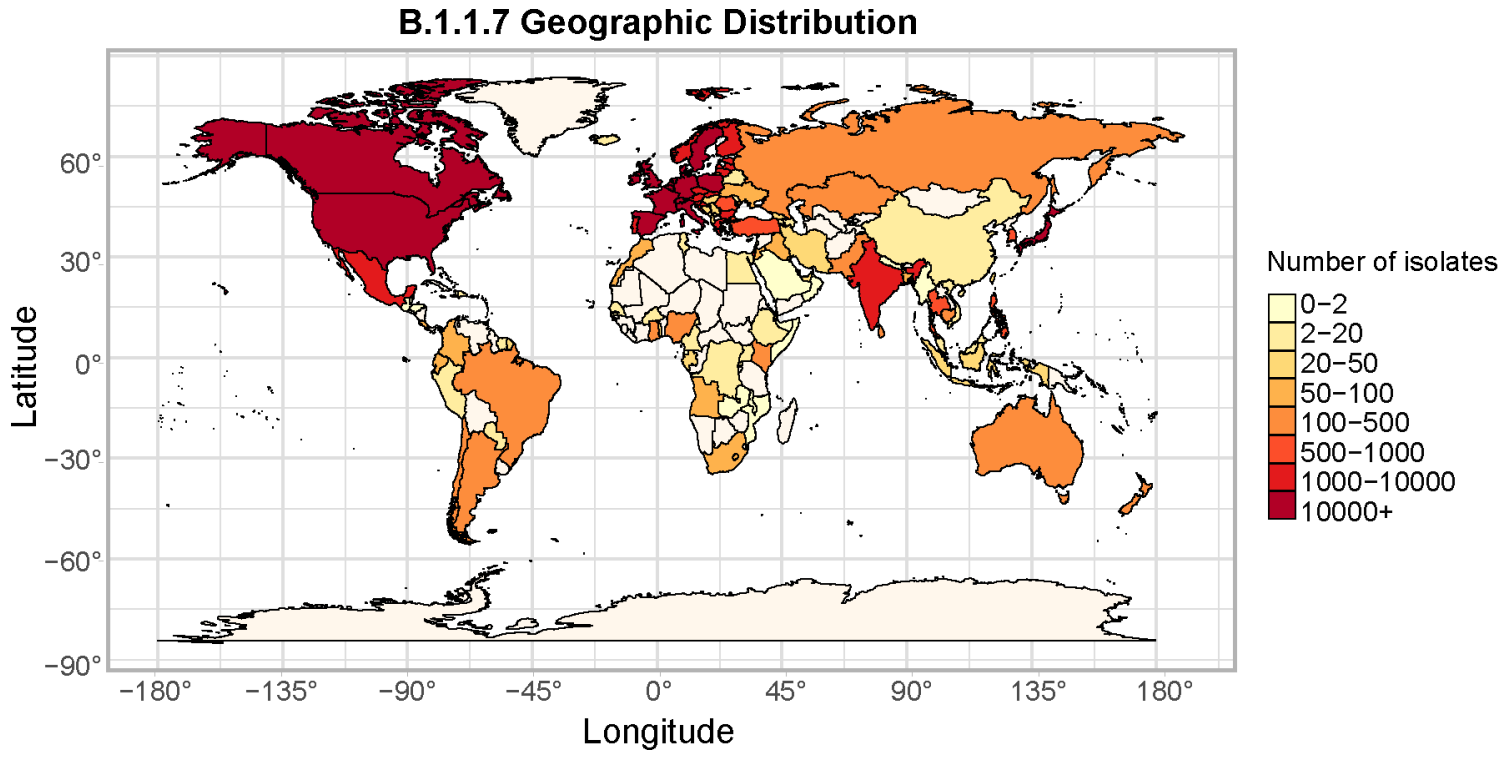

618 Fig. S1. Geographic distribution of the B.1.1.7 variant. Heatmap of the geographic

619 distribution of B.1.1.7 isolates retrieved from the GISAID EpiCoV database as of 28th June, 6202021. 
629

630

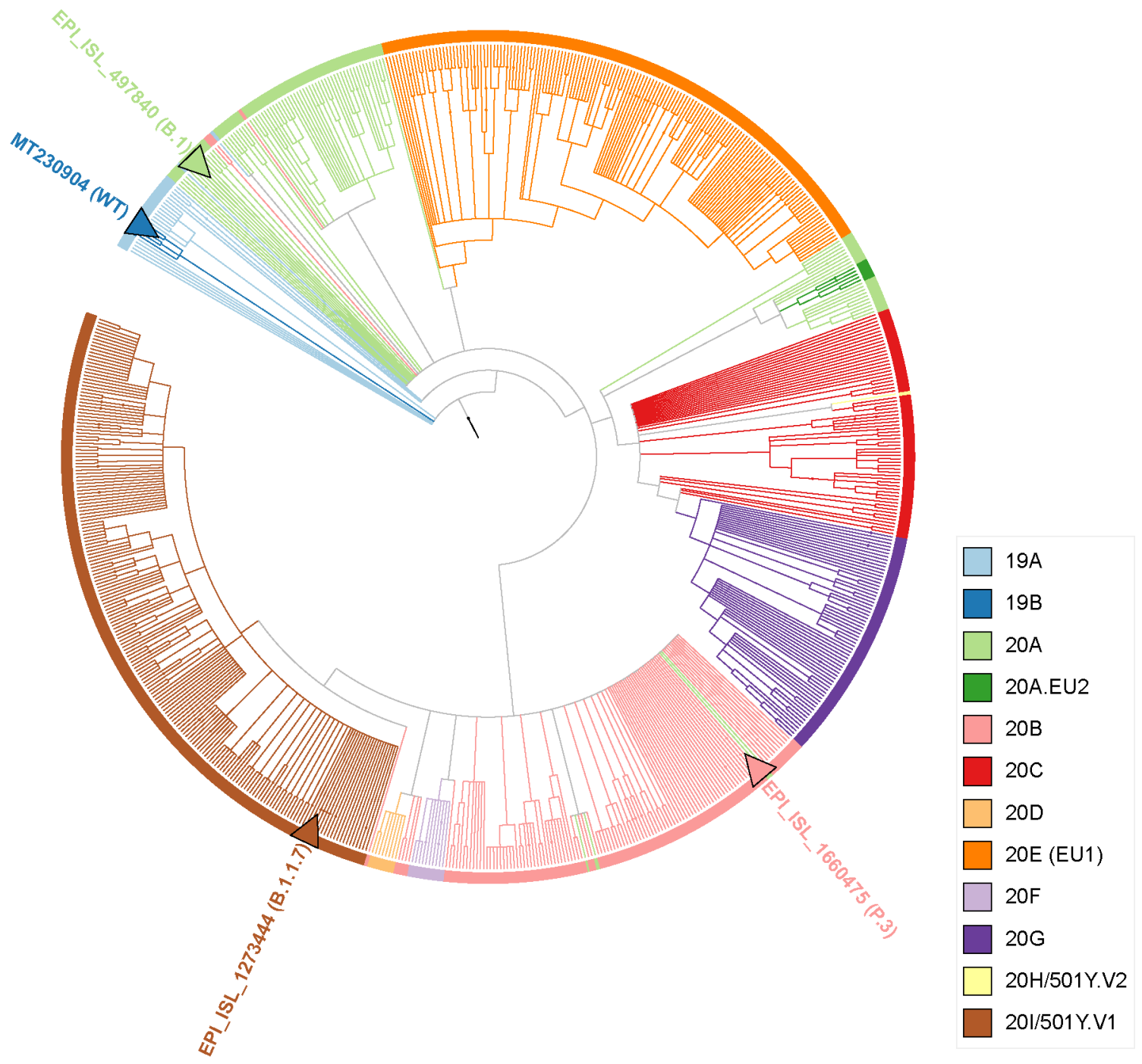

Fig. S2. Phylogenetic tree of virus strains used in this study. Phylogenetic tree showing evolutionary position of the wild type SARS-CoV-2 (Genbank: MT230904), SARS-CoV-2 variant B.1 (GISAID: EPI_ISL_497840), B.1.1.7 (GISAID: EPI_ISL_1273444), and P.3 (GISAID: EPI_ISL_1660475) as of $15^{\text {th }}$ April, 2021. To place the four SARS-CoV-2 sequences in the existing phylogenetic tree, full-length sequences were uploaded to UshER with default parameters. The phylogenetic tree including genomes from GeneBank, COG-UK and CNCB as of April 15, 2021. Phylogenetic tree follow-up modification was done in iTOL. 

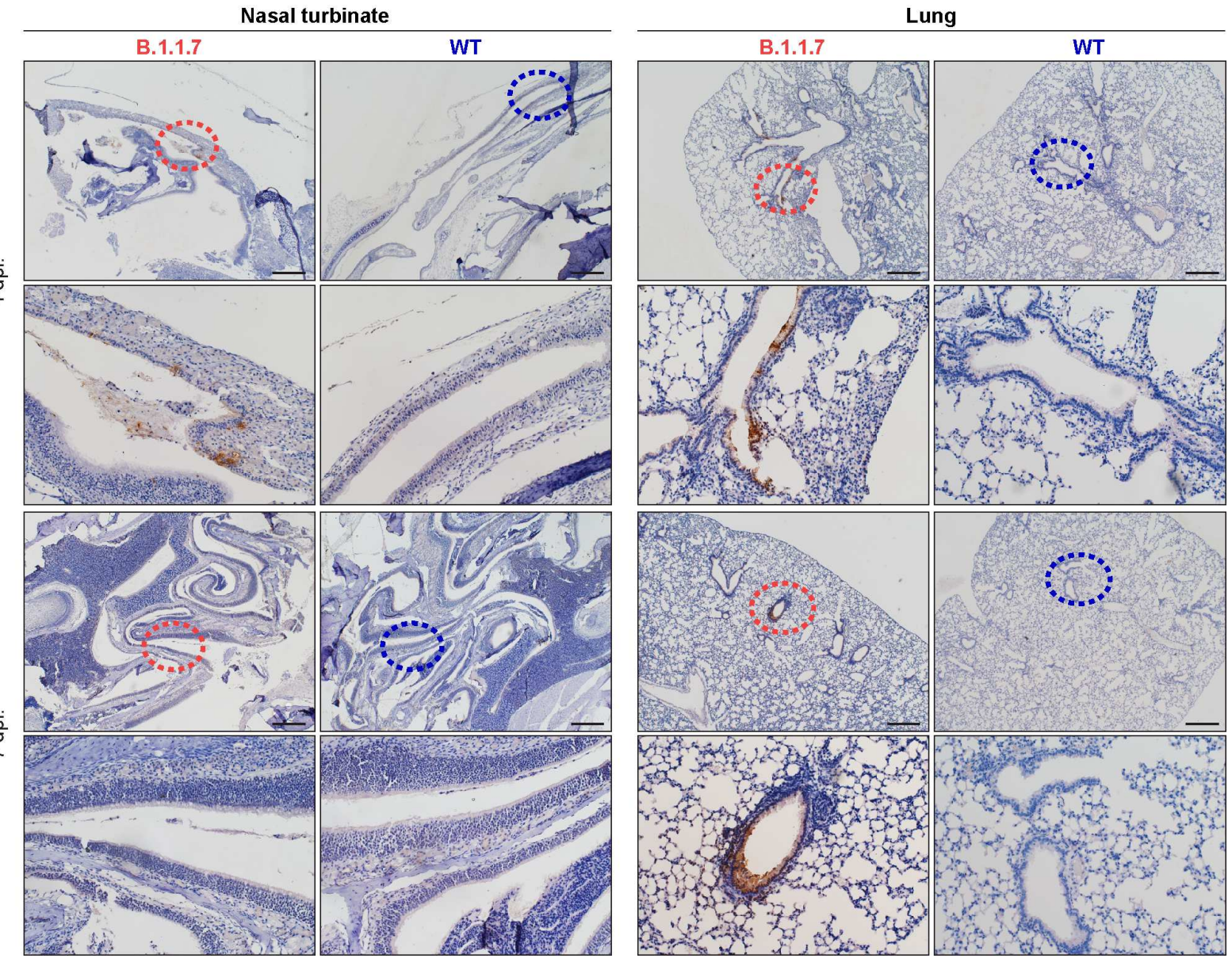

Fig. S3. Immunohistochemistry staining of nasal turbinates and lungs from SARS-CoV-

2-inocuated C57B6 mice. Representative images of immunohistochemistry staining for the detection of SARS-CoV-2 nucleocapsid protein (brown) in the nasal turbinates (left) and lung (right) from B.1.1.7- or WT SARS-CoV-2-inoculated mice on 4 d.p.i. and 7 d.p.i.. Insets were shown for the circled areas. Scale bar, $500 \mu \mathrm{m}$. 
a

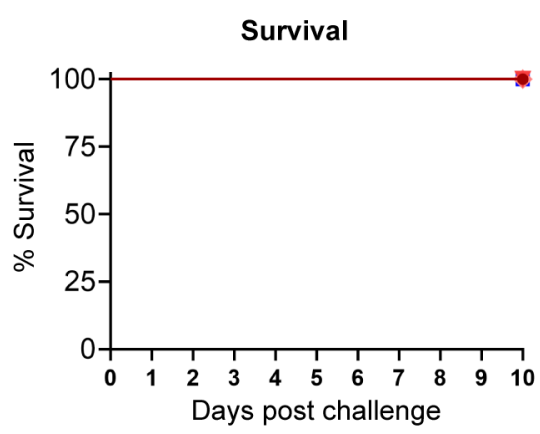

b

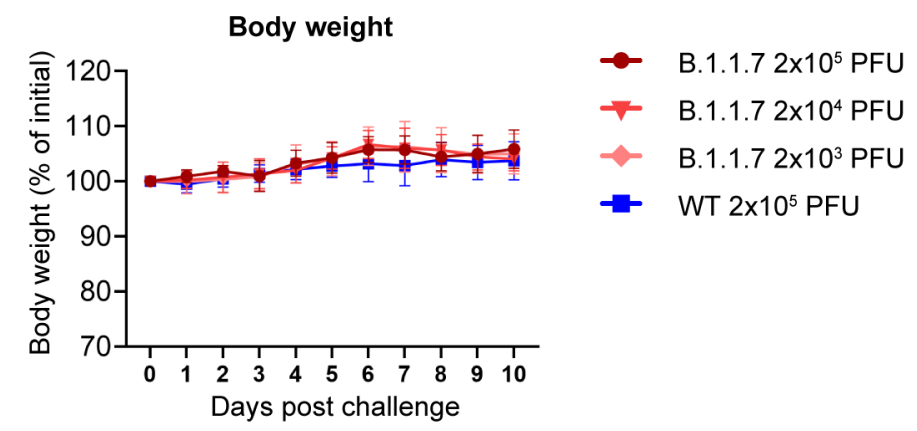

654 Fig. S4. Survival and body weight of C57B6 mice infected with B.1.1.7 variant. C57B6

655 mice were intranasally inoculated with $2 \times 10^{5}$ PFU WT SARS-CoV-2 or with B.1.1.7 at

$6562 \times 10^{3}, 2 \times 10^{4}$ or $2 \times 10^{5}$ PFU. (a) Mice survival and (b) body weight changes were shown until $657 \quad 10$ d.p.i..

658 
a

\begin{tabular}{|c|c|c|c|c|c|c|c|c|c|c|c|c|c|c|c|c|c|c|c|c|}
\hline \multicolumn{21}{|c|}{ Sequence variation of ACE2 residues interacting with SARS-CoV-2 spike RBD } \\
\hline Species & 24 & 27 & 28 & 30 & 31 & 34 & 35 & 37 & 38 & 41 & 42 & 79 & 82 & 83 & 330 & 353 & 354 & 355 & 357 & 393 \\
\hline Homo sapiens & Q & $\mathrm{T}$ & $\mathrm{F}$ & D & $\mathrm{K}$ & $\mathrm{H}$ & $\mathrm{E}$ & $\mathrm{E}$ & $\mathrm{D}$ & $\mathrm{Y}$ & Q & $\mathrm{L}$ & M & Y & $\mathrm{N}$ & $\mathrm{K}$ & G & $\mathrm{D}$ & $\mathrm{R}$ & $\mathrm{R}$ \\
\hline Mus musculus & $\mathrm{N}$ & & . & $\mathrm{N}$ & $\mathrm{N}$ & Q & & . & . & & & T & $\mathrm{S}$ & $\mathrm{F}$ & & $\mathrm{H}$ & . & . & . & \\
\hline Rattus norvegicus & $\mathrm{K}$ & $\mathrm{S}$ & . & $\mathrm{N}$ & . & Q & . & . & . & . & . & 1 & $\mathrm{~N}$ & $\mathrm{~F}$ & . & $\mathrm{H}$ & . & . & . & \\
\hline
\end{tabular}

b

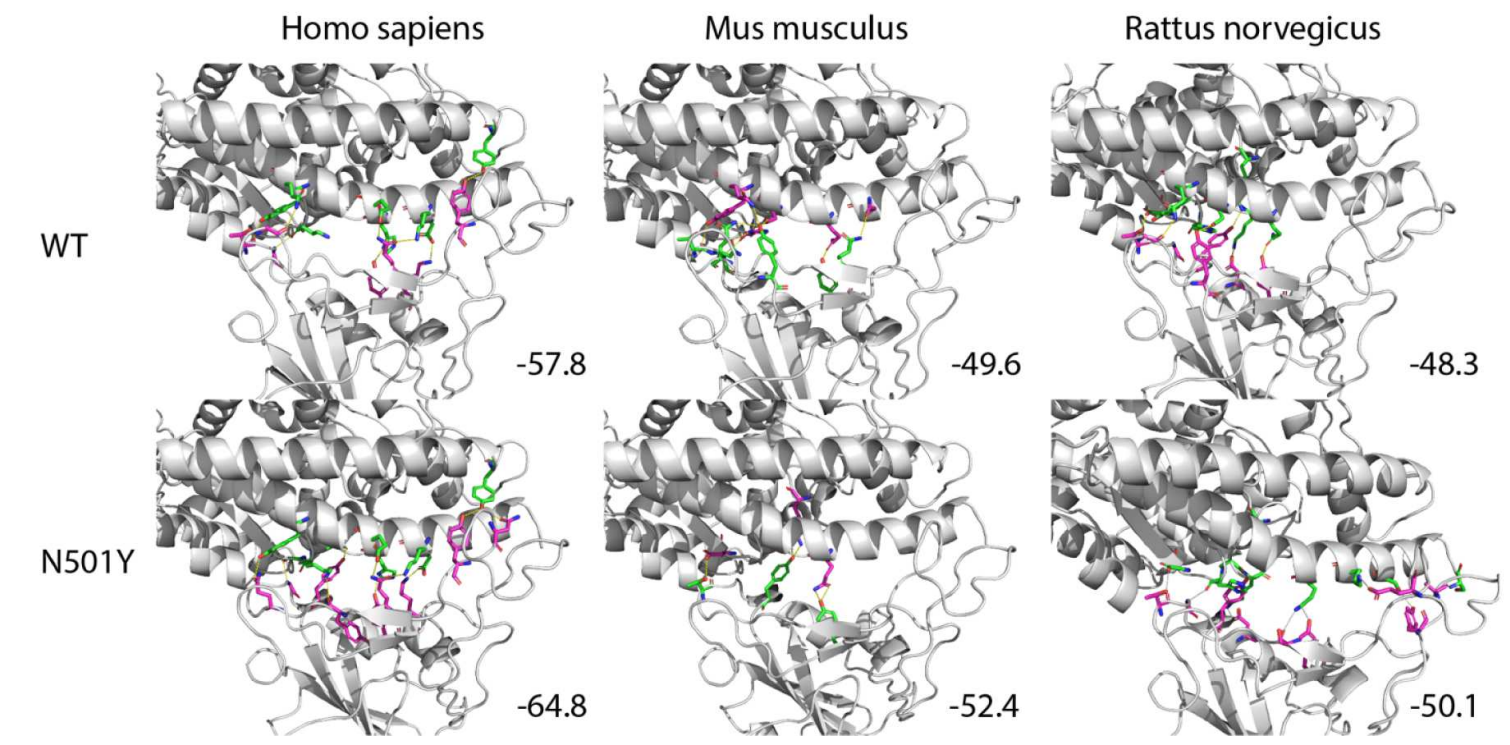

660 Fig. S5. Interaction between SARS-CoV-2 spike RBD and ACE2. (a) Sequence alignment

661 of the interface residues from ACE2 proteins of human, mouse, and rat that are within $4.0 \AA$

662 of RBD. The dots indicate amino acid residues that are identical to that of human. (b)

663 Binding mode and energy of ACE2 proteins from different species with wild type and

664 N501Y RBD. ACE2 and RBD were shown in gray cartoon representation. Polar interactions

665 were indicated with yellow dotted lines. Polar interaction partners from ACE2 and RBD were

666 shown as green and magenta sticks. The predicted binding affinities were labelled 667 accordingly. 


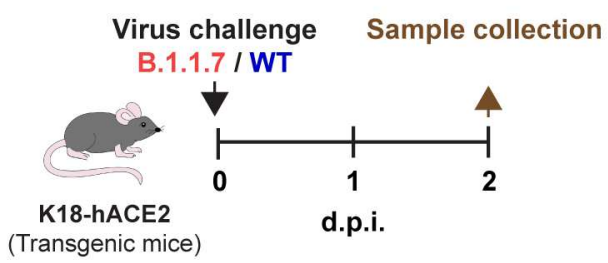

b

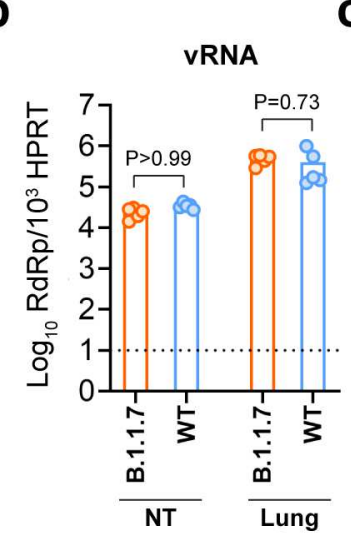

C

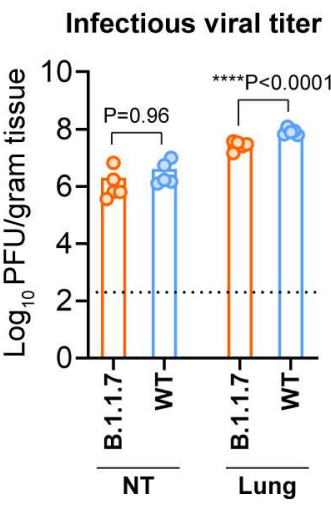

d

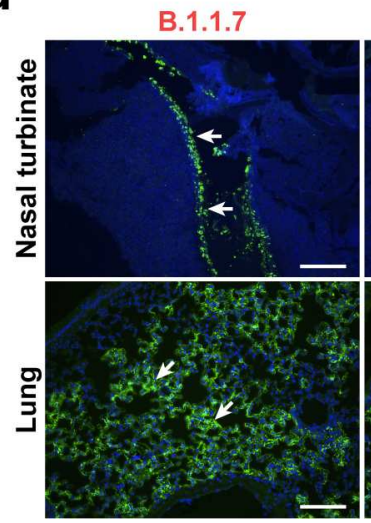

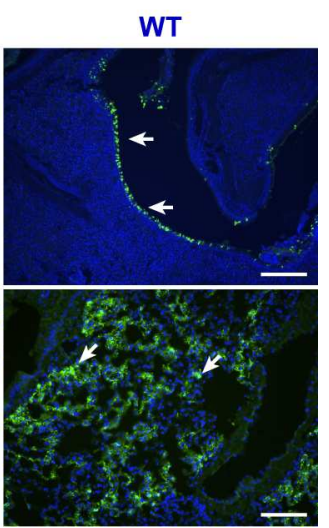

670 Fig. S6. B.1.1.7 and WT SARS-CoV-2 infection in K18-hACE2 mice. (a) Schematic

671 illustration of the infection experiment. (b) Virus genome copy quantified with RT-qPCR in

672 the nasal turbinate and lung. NT, nasal turbinate. (c) Infectious virus titer quantified with

673 plaque assays in the nasal turbinate and lung. (d) Representative images of

674 immunofluorescence staining to show the expression of nucleocapsid protein (green, white

675 arrows) in the lung and nasal turbinates. Scale bar, $200 \mu \mathrm{m}$. Statistical differences were

676 determined with two-tailed Student's t-test in (b) and (c). * represented $\mathrm{P}<0.05$; **

677 represented $\mathrm{P}<0.01 ; * * *$ represented $\mathrm{P}<0.001 ; * * * *$ represented $\mathrm{P}<0.0001$. NT, nasal

678 turbinates. 